Supporting Information for:

\title{
Nickel-Catalyzed Formal Aminocarbonylation of Secondary Benzyl Chlorides with Isocyanides
}

Yun Wang, Wenyi Huang, Chenglong Wang, Jingping $Q u^{*}$, and Yifeng Chen*

Key Laboratory for Advanced Materials and Joint International Research Laboratory of Precision Chemistry and Molecular Engineering, Feringa Nobel Prize Scientist Joint Research Center, Frontiers Science Center for Materiobiology and Dynamic Chemistry, School of Chemistry and Molecular Engineering, East China University of Science \& Technology, 130 Meilong Road, Shanghai, 200237, China.

E-mail: qujp@dlut.edu.cn; yifengchen@ecust.edu.cn 


\section{Table of Contents}

General Information.

S-3

Synthesis and Characterization of New Substrates............................................

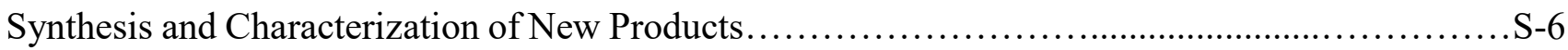

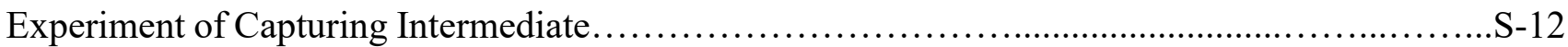

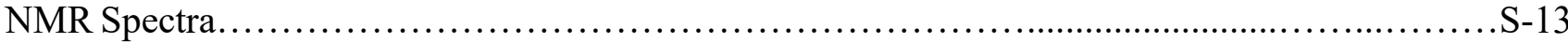

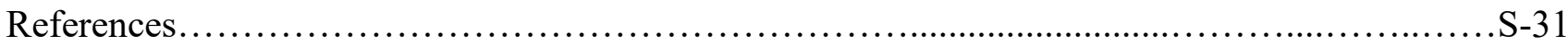




\section{General information}

All reactions were carried out under nitrogen atmosphere and anhydrous conditions unless otherwise indicated. All manipulations of air-sensitive or moisture-sensitive compounds were performed in a glovebox under an atmosphere of nitrogen. Unless otherwise noted, all catalytic reactions were run in dried glassware. Toluene and THF were distilled from sodium/benzophenone. DCM was distilled over $\mathrm{CaH}_{2}$. Ni(COD) $)_{2}$ (CAS 1295-35-8) was purchased from Sinocompound; IMes $\bullet \mathrm{HCl}$ was purchased from Energy Chemical; ${ }^{t} \mathrm{BuNC}(7188-38-7)$ was purchased from Meryer. Reactions were monitored by thin-layer chromatography (TLC) carried out on $0.20 \mathrm{~mm}$ Huanghai silica gel plates (HSGF 254) using UV light as the visualizing agent. All new compounds were characterized by means of GC, GC-MS, ${ }^{1} \mathrm{H}-\mathrm{NMR},{ }^{13} \mathrm{C}-\mathrm{NMR}$, and HR-MS. GC analysis was performed on Agilent Technologies 7820A GC system. GC-MS analysis was performed on Agilent Technologies 7820A, 5977B GC-MS system. GC/GC-MS runs were performed with the following method: GC; HP5 column; inlet temperature $250{ }^{\circ} \mathrm{C}$; column temperature $100{ }^{\circ} \mathrm{C}$ for $1 \mathrm{~min}$, then $50{ }^{\circ} \mathrm{C} / \mathrm{min}$ to $280{ }^{\circ} \mathrm{C}$, then $280{ }^{\circ} \mathrm{C}$ for $6 \mathrm{~min}$. NMR spectra were recorded using a Bruker AVANCE III $400 \mathrm{MHz}$ NMR spectrometer and can be found at the end of the paper. High-resolution mass spectra (HRMS) were recorded on a Waters GCT Premier mass spectrometer using EI-TOF (electron ionization-time of flight) or Waters XEVO G2 TOF mass spectrometer using ESI-TOF (electrospray ionization-time of flight). All ${ }^{1} \mathrm{H}-\mathrm{NMR}$ data are reported in $\delta$ units, parts per million (ppm), and were calibrated relative to the signals for residual chloroform $(7.26 \mathrm{ppm})$ in deuterochloroform $\left(\mathrm{CDCl}_{3}\right)$. All ${ }^{13} \mathrm{C}-\mathrm{NMR}$ data are reported in ppm relative to $\mathrm{CDCl}_{3}(77.16 \mathrm{ppm})$ and were obtained with ${ }^{1} \mathrm{H}$ decoupling. The following abbreviations or combinations thereof were used to explain the multiplicities: $\mathrm{s}=$ singlet, $\mathrm{d}$ $=$ doublet, $\mathrm{t}=$ triplet, $\mathrm{q}=$ quartet, $\mathrm{br}=$ broad, $\mathrm{m}=$ multiplet. 


\section{Synthesis and Characterization of New Substrates}

\section{Synthesis of known Substrates}

Compounds $\mathbf{1 a},{ }^{[1]} \mathbf{1 b},{ }^{[2]} \mathbf{1 e},{ }^{[3]} \mathbf{1 f},{ }^{[4]} \mathbf{1 g},{ }^{[4]} \mathbf{1 h},{ }^{[5]} \mathbf{1 i},{ }^{[6]} \mathbf{1 j},{ }^{[7]} \mathbf{1 k},{ }^{[8]} \mathbf{1 m},{ }^{[9]} \mathbf{1 o},{ }^{[10]} \mathbf{1 s},{ }^{[11]} \mathbf{2 b},{ }^{[12]} \mathbf{2 c},{ }^{[13]}$ $\mathbf{2 d},{ }^{[13]} \mathbf{2 e},{ }^{[13]} \mathbf{2 \mathbf { f } ^ { [ 1 4 ] }}$ were synthesized according to the published procedures. Compounds $\mathbf{1 d}, \mathbf{1 n}, \mathbf{2 a}$, $\mathbf{2 g}$ are commercially available.

\section{Synthesis of New Substrates}

\section{2-(chloro(cyclohexyl)methyl)naphthalene (1c)}

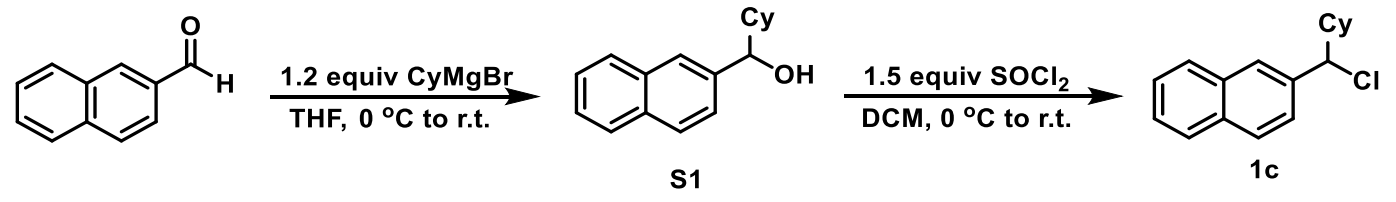

To a solution of 2-naphthaldehyde (1.0 equiv, $10 \mathrm{mmol}, 1.6 \mathrm{~g})$ in anhydrous THF (0.5 M, $20 \mathrm{~mL})$ at $0{ }^{\circ} \mathrm{C}$ was slowly added $\mathrm{CyMgBr}(0.34 \mathrm{M}, 1.2$ equiv, $12 \mathrm{mmol}, 35 \mathrm{~mL})$, and then the resulting mixture was stirred at $0{ }^{\circ} \mathrm{C}$ for $30 \mathrm{~min}$ and then was allowed to stir at room temperature until complete by TLC analysis. When the starting material was consumed, the reaction was quenched with sat. aq. $\mathrm{NH}_{4} \mathrm{Cl}$ and extracted three times with ethyl acetate. The combined organic layer was washed with brine, dried over $\mathrm{Na}_{2} \mathrm{SO}_{4}$, filtered and concentrated in vacuo. The resulting residue was purified by flash column chromatography on silica gel to afford $\mathbf{S 1}$ as a white solid $(1.20 \mathrm{~g}, 52 \%), \mathbf{R}_{\mathbf{f}}=0.39(\mathrm{PE} / \mathrm{EtOAc}=10 / 1)$.

To a solution of $\mathbf{S 1}(1.0$ equiv, $5 \mathrm{mmol}, 1.20 \mathrm{~g})$ in DCM $(0.5 \mathrm{M}, 10 \mathrm{~mL})$ at $0{ }^{\circ} \mathrm{C}$ was slowly added $\mathrm{SOCl}_{2}(1.5$ equiv, 7.5 equiv, $456.0 \mu \mathrm{L})$, and then the reaction mixture was stirred at room temperature until complete by TLC analysis. When the starting material was consumed, the reaction was quenched with sat. aq. $\mathrm{NaHCO}_{3}$ and extracted three times with DCM. The combined organic layer was washed with brine, dried over $\mathrm{Na}_{2} \mathrm{SO}_{4}$, and then filtered. The filtrate was concentrated in vacuo and the residue was purified by flash column chromatography on silica gel (PE) to afford 1c as a white solid (561.8 $\mathrm{mg}, 44 \%) . \mathbf{R} \mathbf{f}=0.61(\mathrm{PE}) ;{ }^{1} \mathbf{H}$ NMR $\left(400 \mathrm{MHz}, \mathrm{CDCl}_{3}\right): \delta 7.86-7.82(\mathrm{~m}, 3 \mathrm{H}), 7.73(\mathrm{~s}, 1 \mathrm{H}), 7.53-7.46$ $(\mathrm{m}, 3 \mathrm{H}), 4.78(\mathrm{~d}, J=8.8 \mathrm{~Hz}, 1 \mathrm{H}), 2.28(\mathrm{~d}, J=12.8 \mathrm{~Hz}, 1 \mathrm{H}), 2.06-1.95(\mathrm{~m}, 1 \mathrm{H}), 1.84-1.80(\mathrm{~m}, 1 \mathrm{H})$, $1.67-1.64(\mathrm{~m}, 2 \mathrm{H}), 1.46-1.43(\mathrm{~m}, 1 \mathrm{H}), 1.35-1.22(\mathrm{~m}, 1 \mathrm{H}), 1.19-1.07(\mathrm{~m}, 3 \mathrm{H}), 0.99-0.89(\mathrm{~m}, 1 \mathrm{H}) ;{ }^{13} \mathrm{C}$ NMR (100 MHz, $\left.\mathrm{CDCl}_{3}\right): \delta 138.4,133.2,133.0,128.5,128.1,127.8,126.8,126.5,126.4,125.2,70.3$, 45.7, 30.7, 30.6, 26.3, 26.1, 26.0; HRMS (EI): Calcd for $\mathrm{C}_{17} \mathrm{H}_{19} \mathrm{Cl}$ : 258.1175; found: 258.1169 . 
2-(1-chloroethyl)pyrazine (11)
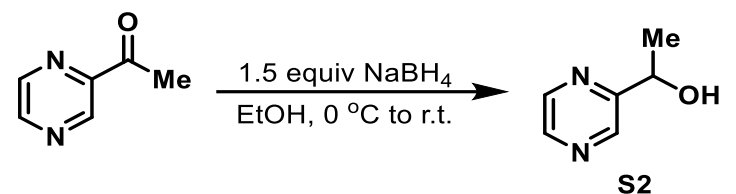

$\underset{\mathrm{CHCl}_{3}, 0^{\circ} \mathrm{C} \text { to r.t. }}{\stackrel{1.05 \text { equiv } \mathrm{SOCl}_{2}}{\longrightarrow}}$<smiles>CC(Cl)c1cnccn1</smiles>

11

To a solution of 1-pyrazinylethanone (1.0 equiv) dissolved in ethyl alcohol (1.0 M) was added sodium borohydride ( 1.5 equiv) in small portions at $0{ }^{\circ} \mathrm{C}$. Then the resulting mixture was allowed to stir at room temperature until complete by TLC analysis. When the starting material was consumed, the reaction was quenched with water and extracted three times with ethyl acetate. The combined organic layer was washed with brine, dried over $\mathrm{Na}_{2} \mathrm{SO}_{4}$, filtered and concentrated in vacuo. The resulting residue was purified by flash column chromatography on silica gel to afford $\mathbf{S 2}$.

To a solution of $\mathbf{S 2}$ (1.0 equiv) in chloroform $(0.6 \mathrm{M})$ at $0{ }^{\circ} \mathrm{C}$ was slowly added thionyl chloride (1.05 equiv), and then the reaction mixture was stirred at room temperature until complete by TLC analysis. When the starting material was consumed, the reaction was quenched with sat. aq. $\mathrm{NaHCO}_{3}$ and extracted three times with DCM. The combined organic layer was washed with brine, dried over $\mathrm{Na}_{2} \mathrm{SO}_{4}$, and then filtered. The filtrate was concentrated in vacuo and the residue was purified by flash column chromatography on silica gel to afford $\mathbf{1 l}$ as a brown oil (over two steps, $29 \%$ ); $\mathbf{R}_{\mathbf{f}}=0.36$ $(\mathrm{PE} / \mathrm{EtOAc}=5 / 1) ;{ }^{1} \mathbf{H}$ NMR $\left(400 \mathrm{MHz}, \mathrm{CDCl}_{3}\right): \delta 8.76(\mathrm{~s}, 1 \mathrm{H}), 8.54-8.51(\mathrm{~m}, 2 \mathrm{H}), 5.15(\mathrm{q}, J=6.8$ $\mathrm{Hz}, 1 \mathrm{H}), 1.90(\mathrm{~d}, J=6.8 \mathrm{~Hz}, 3 \mathrm{H}) ;{ }^{13} \mathbf{C} \mathbf{N M R}\left(100 \mathrm{MHz}, \mathrm{CDCl}_{3}\right): \delta 156.3,144.1,143.9,143.4,56.1$, 24.3. HRMS (ESI): Calcd for $\mathrm{C}_{6} \mathrm{H}_{8} \mathrm{ClN}_{2}[\mathrm{M}+\mathrm{H}]^{+}$: 143.0376; found: 143.0374 . 


\section{Synthesis and Characterization of New Products}

\section{General procedure for Ni-catalyzed aminocarbonylation of secondary benzyl chlorides.}

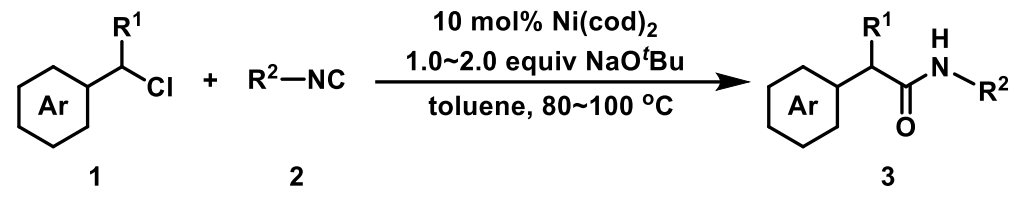

In air, sodium tert-butoxide (1.0 2.0 equiv) was added to an over-dried Schlenk tube equipped with a stir bar. The vessel was moved to the glovebox, $\mathrm{Ni}(\mathrm{COD})_{2}(10 \mathrm{~mol} \%)$ was added. Then, the vessel was taken out of the glovebox before toluene $(0.1 \mathrm{M})$, benzyl chloride 1 (1.0 equiv) and isocyanide 2 (1.1 1.5 equiv) were added in turn by syringe. The reaction mixture was stirred at oil bath until complete by TLC analysis. When the starting material was consumed, the reaction was quenched with $1 \mathrm{M}$ aq. $\mathrm{HCl}$ (if substrates contain heterocycle, sat. aq. $\mathrm{NaHCO}_{3}$ was added until $\mathrm{pH}>$ 7) and extracted three times with ethyl acetate. The combined organic layer was dried over $\mathrm{Na}_{2} \mathrm{SO}_{4}$, and then filtered. The filtrate was concentrated in vacuo and the residue was purified by flash column chromatography.

\section{Procedure for the Synthesis of the Amide 3d on a 1.0 mmol-Scale}

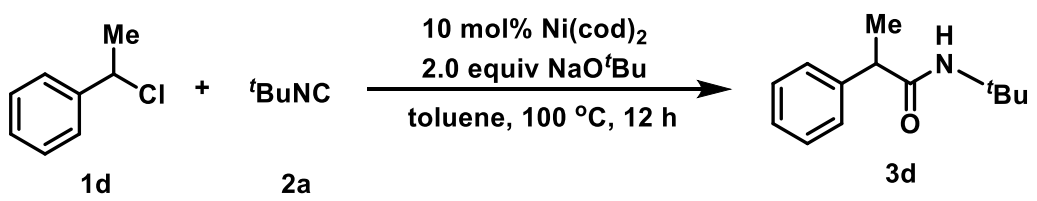

In air, sodium tert-butoxide (2.0 equiv, $2.0 \mathrm{mmol}, 192 \mathrm{mg}$ ) was added to an over-dried Schlenk tube equipped with a stir bar. The vessel was moved to the glovebox, $\mathrm{Ni}(\mathrm{COD})_{2}(10 \mathrm{~mol} \%, 0.1 \mathrm{mmol}$, $27.5 \mathrm{mg}$ ) was added. Then, the vessel was taken out of the glovebox before toluene $(0.1 \mathrm{M}, 10 \mathrm{~mL})$, benzyl chloride $1 \mathrm{~d}(1.0$ equiv, $1.0 \mathrm{mmol}, 134 \mu \mathrm{L})$ and tert-butyl isocyanide (1.5 equiv, $1.5 \mathrm{mmol}, 170$ $\mu \mathrm{L})$ were added in turn by syringe. The reaction mixture was stirred at $100{ }^{\circ} \mathrm{C}$ oil bath until complete by TLC analysis. When the starting material was consumed, the reaction was quenched with $1 \mathrm{M}$ aq. $\mathrm{HCl}$ and extracted three times with ethyl acetate. The combined organic layer was dried over $\mathrm{Na}_{2} \mathrm{SO}_{4}$, and then filtered. The filtrate was concentrated in vacuo and the residue was purified by flash column chromatography on silica gel to afford $\mathbf{3 d}$ as a light yellow solid $(163.4 \mathrm{mg}, 80 \%)$. $\mathbf{R}_{\mathbf{f}}=0.48$ (PE/EtOAc $=5 / 1) ;{ }^{1} \mathbf{H}$ NMR (400 MHz, $\left.\mathrm{CDCl}_{3}\right): \delta 7.36-7.32(\mathrm{~m}, 2 \mathrm{H}), 7.29-7.24(\mathrm{~m}, 3 \mathrm{H}), 5.15$ (bs, 1H), 3.47 (q, $J=7.2 \mathrm{~Hz}, 1 \mathrm{H}), 1.48(\mathrm{~d}, J=7.2 \mathrm{~Hz}, 3 \mathrm{H}), 1.27(\mathrm{~s}, 9 \mathrm{H}) ;{ }^{13} \mathbf{C} \mathbf{N M R}\left(100 \mathrm{MHz}, \mathrm{CDCl}_{3}\right): \delta 173.7,142.1$, $129.0,127.6,127.2,51.3,47.9,28.8,18.8$. The spectral data is consistent with previously reported literature values. ${ }^{[15]}$ 


\section{Characterization of Formal Aminocarbonylation Products}

\section{$N$-(tert-butyl)-2-(naphthalen-2-yl)propanamide (3a)}

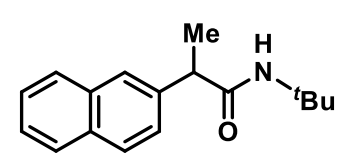

General procedure was followed on $0.5 \mathrm{mmol}$ scale with 2.0 equiv sodium tertbutoxide and 1.5 equiv tert-butyl isocyanide at $100{ }^{\circ} \mathrm{C}$ for 5 hours. The reaction mixture was purification by flash column chromatography on silica gel $(\mathrm{PE} /$ EtOAc $=10 / 1-5 / 1)$ afforded 3a as a light yellow solid $(97.9 \mathrm{mg}, 77 \%)$. $\mathbf{R}_{\mathbf{f}}$ $=0.41(\mathrm{PE} / \mathrm{EtOAc}=5 / 1) ;{ }^{1} \mathbf{H}$ NMR $\left(400 \mathrm{MHz}, \mathrm{CDCl}_{3}\right): \delta 7.84-7.82(\mathrm{~m}, 3 \mathrm{H}), 7.72(\mathrm{~s}, 1 \mathrm{H}), 7.51-7.44$ $(\mathrm{m}, 2 \mathrm{H}), 7.41(\mathrm{dd}, J=8.4,1.6 \mathrm{~Hz}, 1 \mathrm{H}), 5.18(\mathrm{bs}, 1 \mathrm{H}), 3.63(\mathrm{q}, J=7.2 \mathrm{~Hz}, 1 \mathrm{H}), 1.56(\mathrm{~d}, J=7.2 \mathrm{~Hz}$, $3 \mathrm{H}), 1.26(\mathrm{~s}, 9 \mathrm{H}) ;{ }^{13} \mathbf{C}$ NMR $\left(100 \mathrm{MHz}, \mathrm{CDCl}_{3}\right): \delta 173.5,139.6,133.7,132.7,128.8,127.9,127.8$, 126.4, 126.2 126.0, 125.8, 51.3, 48.1, 28.8, 18.9; HRMS (EI): Calcd for $\mathrm{C}_{17} \mathrm{H}_{21} \mathrm{NO}$ : 255.1623; found: 255.1622 .

\section{$N$-(tert-butyl)-2-(naphthalen-2-yl)butanamide (3b)}

General procedure was followed on $0.5 \mathrm{mmol}$ scale with 1.5 equiv sodium tert-
butoxide and 1.5 equiv tert-butyl isocyanide at $100^{\circ} \mathrm{C}$ for 9.5 hours. The reaction
mixture was purification by flash column chromatography on silica gel $(\mathrm{PE} / \mathrm{EtOAc}=20 / 1-5 / 1)$ afforded $\mathbf{3 b}$ as a light yellow solid $(113.2 \mathrm{mg}, 84 \%) . \mathbf{R}_{\mathbf{f}}=0.59(\mathrm{PE} / \mathrm{EtOAc}=$ 5/1); ${ }^{1}$ H NMR $\left(400 \mathrm{MHz}, \mathrm{CDCl}_{3}\right): \delta$ 7.84-7.82 (m, 3H), $7.75(\mathrm{~s}, 1 \mathrm{H}), 7.51-7.44(\mathrm{~m}, 3 \mathrm{H}), 5.42(\mathrm{bs}$, $1 \mathrm{H}), 3.33$ (t, $J=7.6 \mathrm{~Hz}, 1 \mathrm{H}), 2.31-2.20(\mathrm{~m}, 1 \mathrm{H}), 1.92-1.81(\mathrm{~m}, 1 \mathrm{H}), 1.30(\mathrm{~s}, 9 \mathrm{H}), 0.93$ (t, $J=7.2 \mathrm{~Hz}$, 3H); ${ }^{13} \mathbf{C}$ NMR $\left(100 \mathrm{MHz}, \mathrm{CDCl}_{3}\right): \delta 172.8,138.2,133.5,132.6,128.4,127.8,127.7,126.7,126.1$, 125.9, 125.7, 55.9, 51.2, 28.8, 26.6, 12.5; HRMS (EI): Calcd for $\mathrm{C}_{18} \mathrm{H}_{23} \mathrm{NO}$ : 269.1780; found: 269.1782.

\section{$N$-(tert-butyl)-2-cyclohexyl-2-(naphthalen-2-yl)acetamide (3c)}<smiles></smiles>

General procedure was followed on $0.5 \mathrm{mmol}$ scale with 2.0 equiv sodium tertbutoxide and 1.5 equiv tert-butyl isocyanide at $100{ }^{\circ} \mathrm{C}$ for 10 hours and then

$120{ }^{\circ} \mathrm{C}$ for $5 \mathrm{~h}$. The reaction mixture was purification by flash column chromatography on silica gel $(\mathrm{PE} / \mathrm{DCM}=1 / 1)$ afforded $\mathbf{3 c}$ as a white solid $(66.0 \mathrm{mg}, 41 \%) . \mathbf{R}_{\mathbf{f}}=0.50$ $(\mathrm{PE} / \mathrm{EtOAc}=10 / 1) ;{ }^{1} \mathbf{H}$ NMR $\left(400 \mathrm{MHz}, \mathrm{CDCl}_{3}\right): \delta 7.82-7.78(\mathrm{~m}, 3 \mathrm{H}), 7.73(\mathrm{~s}, 1 \mathrm{H}), 7.54(\mathrm{dd}, J=8.4$, $1.2 \mathrm{~Hz}, 1 \mathrm{H}), 7.48-7.41(\mathrm{~m}, 2 \mathrm{H}), 5.37$ (bs, 1H), $2.94(\mathrm{~d}, J=10.0 \mathrm{~Hz}, 1 \mathrm{H}), 2.16$ (qt, $J=11.2,3.2 \mathrm{~Hz}$, $1 \mathrm{H}), 2.00(\mathrm{~d}, J=12.8 \mathrm{~Hz}, 1 \mathrm{H}), 1.76-1.73(\mathrm{~m}, 1 \mathrm{H}), 1.65-1.56(\mathrm{~m}, 2 \mathrm{H}), 1.40-1.31(\mathrm{~m}, 2 \mathrm{H}), 1.28(\mathrm{~s}$, 9H), 1.19-1.10 (m, 2H), 1.03 (qd, $J=11.2,3.2 \mathrm{~Hz}, 1 \mathrm{H}), 0.75(\mathrm{qd}, J=12.0,3.2 \mathrm{~Hz}, 1 \mathrm{H}) ;{ }^{13} \mathbf{C}$ NMR $\left(100 \mathrm{MHz} \mathrm{CDCl}_{3}\right): \delta 172.5,137.2,133.5,132.7,128.1,127.9,127.7,127.2,126.5,126.0,125.6,61.8$, 51.5, 40.9, 32.3, 31.0, 28.9, 26.6, 26.3, 26.3; HRMS (EI): Calcd for $\mathrm{C}_{22} \mathrm{H}_{29} \mathrm{NO}$ : 323.2249; found: 323.2248 .

\section{$N$-(tert-butyl)-3-methyl-2-phenylbutanamide (3e)}

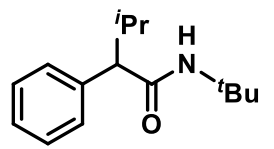

General procedure was followed on $0.5 \mathrm{mmol}$ scale with 1.1 equiv sodium tertbutoxide and 1.1 equiv tert-butyl isocyanide at $100{ }^{\circ} \mathrm{C}$ for $22 \mathrm{~h}$. The reaction mixture was purification by flash column chromatography on silica gel $(\mathrm{PE} / \mathrm{EtOAc}=20 / 1-$ $10 / 1)$ afforded $\mathbf{3 e}$ as a white solid $(60.4 \mathrm{mg}, 52 \%) . \mathbf{R}_{\mathbf{f}}=0.45(\mathrm{PE} / \mathrm{EtOAc}=10 / 1) ;{ }^{1} \mathbf{H ~ N M R}(400 \mathrm{MHz}$, 
$\left.\mathrm{CDCl}_{3}\right): \delta 7.37-7.35(\mathrm{~m}, 2 \mathrm{H}), 7.33-7.29(\mathrm{~m}, 2 \mathrm{H}), 7.27-7.23(\mathrm{~m}, 1 \mathrm{H}), 5.42(\mathrm{bs}, 1 \mathrm{H}), 2.74(\mathrm{~d}, J=10.0$ $\mathrm{Hz}, 1 \mathrm{H}), 2.46-2.33(\mathrm{~m}, 1 \mathrm{H}), 1.31(\mathrm{~s}, 9 \mathrm{H}), 1.07(\mathrm{~d}, J=6.8 \mathrm{~Hz}, 3 \mathrm{H}), 0.70(\mathrm{~d}, J=6.8 \mathrm{~Hz}, 3 \mathrm{H}) ;{ }^{13} \mathbf{C} \mathbf{N M R}$ $\left(100 \mathrm{MHz}, \mathrm{CDCl}_{3}\right): \delta 172.8,139.9,128.4,128.3,126.9,62.7,51.3,31.8,28.8,21.7,20.5 ;$ HRMS (EI): Calcd for $\mathrm{C}_{15} \mathrm{H}_{23} \mathrm{NO}$ : 233.1780; found: 233.1778 .

\section{$N$-(tert-butyl)-2-(4-chlorophenyl)propanamide (3f)}

General procedure was followed on $0.5 \mathrm{mmol}$ scale with 2.0 equiv sodium tert-<smiles>O=C([18OH])N[CH]c1ccc(Cl)cc1</smilesbutoxide and 1.5 equiv tert-butyl isocyanide at $100{ }^{\circ} \mathrm{C}$ for $9 \mathrm{~h}$. The reaction mixture was purification by flash column chromatography on silica gel $(\mathrm{PE} / \mathrm{EtOAc}=50 / 1-10 / 1)$ afforded $\mathbf{3 f}$ as a light yellow solid $(81.6 \mathrm{mg}, 68 \%) . \mathbf{R}_{\mathbf{f}}$ $=0.27(\mathrm{PE} / \mathrm{EtOAc}=10 / 1) ;{ }^{1} \mathbf{H}$ NMR $\left(400 \mathrm{MHz}, \mathrm{CDCl}_{3}\right): \delta 7.30(\mathrm{~d}, J=8.4 \mathrm{~Hz}, 2 \mathrm{H}), 7.23(\mathrm{~d}, J=8.8$ $\mathrm{Hz}, 2 \mathrm{H}), 5.27$ (bs, 1H), 3.43 (q, $J=7.2 \mathrm{~Hz}, 1 \mathrm{H}), 1.44(\mathrm{~d}, J=7.2 \mathrm{~Hz}, 3 \mathrm{H}), 1.29(\mathrm{~s}, 9 \mathrm{H}) ;{ }^{13} \mathbf{C}$ NMR $(100$ $\left.\mathrm{MHz}, \mathrm{CDCl}_{3}\right): \delta 173.0,140.6,132.8,129.0,128.9,51.3,47.2,28.7,19.0$. The spectral data is consistent with previously reported literature values. ${ }^{[15]}$

\section{$N$-(tert-butyl)-2-(4-bromophenyl)propanamide (3g)}<smiles>CC(C)NC(=O)C(C)c1ccc(Br)cc1</smiles>

General procedure was followed on $0.2 \mathrm{mmol}$ scale with 1.0 equiv sodium tertbutoxide and 1.1 equiv tert-butyl isocyanide at $80^{\circ} \mathrm{C}$ for $6 \mathrm{~h}$. The reaction mixture was purification by flash column chromatography on silica gel $(\mathrm{PE} / \mathrm{EtOAc}=$ 20/1-10/1) afforded $3 \mathbf{g}$ as a white solid (33.7 $\mathrm{mg}, 59 \%) . \mathbf{R}_{\mathbf{f}}=0.29(\mathrm{PE} / \mathrm{EtOAc}=$

10/1); ${ }^{1} \mathbf{H}$ NMR $\left(400 \mathrm{MHz}, \mathrm{CDCl}_{3}\right): \delta 7.44(\mathrm{~d}, J=8.4 \mathrm{~Hz}, 2 \mathrm{H}), 7.16(\mathrm{~d}, J=8.4 \mathrm{~Hz}, 2 \mathrm{H}), 5.16$ (bs, $1 \mathrm{H}), 3.38(\mathrm{q}, J=7.2 \mathrm{~Hz}, 1 \mathrm{H}), 1.42(\mathrm{~d}, J=7.2 \mathrm{~Hz}, 3 \mathrm{H}), 1.27(\mathrm{~s}, 9 \mathrm{H}) ;{ }^{13} \mathbf{C} \mathbf{N M R}\left(100 \mathrm{MHz}, \mathrm{CDCl}_{3}\right): \delta$ 172.9, 141.1, 132.0, 129.3, 121.0, 51.4, 47.4, 28.8, 19.0; HRMS (EI): Calcd for $\mathrm{C}_{13} \mathrm{H}_{19} \mathrm{BrNO}[\mathrm{M}+$ $\mathrm{H}]^{+}:$284.0645; found: 284.0640 .

\section{$N$-(tert-butyl)-2-(4-iodophenyl)propanamide (3h)}

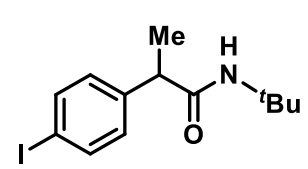

General procedure was followed on $0.5 \mathrm{mmol}$ scale with 1.0 equiv sodium tertbutoxide and 1.1 equiv tert-butyl isocyanide at $90{ }^{\circ} \mathrm{C}$ for $5.5 \mathrm{~h}$. The reaction mixture was purification by flash column chromatography on silica gel $(\mathrm{PE} / \mathrm{EtOAc}=20 / 1-5 / 1)$ afforded $\mathbf{3 h}$ as a white solid $(85.6 \mathrm{mg}, 52 \%) . \mathbf{R}_{\mathbf{f}}=0.28$ $(\mathrm{PE} / \mathrm{EtOAc}=10 / 1) ;{ }^{1} \mathbf{H}$ NMR $\left(400 \mathrm{MHz}, \mathrm{CDCl}_{3}\right): \delta 7.63(\mathrm{~d}, J=8.4 \mathrm{~Hz}, 2 \mathrm{H}), 7.03(\mathrm{~d}, J=8.4 \mathrm{~Hz}, 2 \mathrm{H})$, 5.19 (bs, 1H), 3.36 (q, $J=7.2 \mathrm{~Hz}, 1 \mathrm{H}), 1.41$ (d, $J=7.2 \mathrm{~Hz}, 3 \mathrm{H}), 1.26$ (s, 9H); ${ }^{13} \mathbf{C}$ NMR $(100 \mathrm{MHz}$, $\left.\mathrm{CDCl}_{3}\right): \delta 172.8,141.8,137.9,129.5,92.5,51.3,47.4,28.8,18.9$; HRMS (EI): Calcd for $\mathrm{C}_{13} \mathrm{H}_{18} \mathrm{INO}$ : 331.0433; found: 331.0432 .

\section{$N$-(tert-butyl)-2-(pyridin-2-yl)propanamide (3i)}

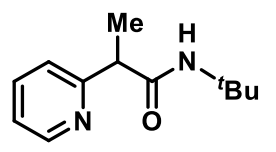

General procedure was followed on $0.5 \mathrm{mmol}$ scale with 2.0 equiv sodium tertbutoxide and 1.5 equiv tert-butyl isocyanide at $100{ }^{\circ} \mathrm{C}$ for $4.5 \mathrm{~h}$. The reaction mixture was purification by flash column chromatography on silica gel $(\mathrm{PE} / \mathrm{EtOAc}=5 / 1-$ $1 / 1)$ afforded $3 \mathbf{i}$ as a black solid $(71.0 \mathrm{mg}, 69 \%) . \mathbf{R}_{\mathbf{f}}=0.31(\mathrm{PE} / \mathrm{EtOAc}=2 / 1) ;{ }^{1} \mathbf{H}$ NMR (400 MHz, $\left.\mathrm{CDCl}_{3}\right): \delta 8.47(\mathrm{dd}, J=4.8,0.4 \mathrm{~Hz}, 1 \mathrm{H}), 7.62(\mathrm{td}, J=7.6,2.0 \mathrm{~Hz}, 1 \mathrm{H}), 7.26(\mathrm{~d}, J=8.0 \mathrm{~Hz}, 1 \mathrm{H}), 7.14$ (ddd, $J=7.6,4.8,1.2 \mathrm{~Hz}, 1 \mathrm{H}), 6.74(\mathrm{bs}, 1 \mathrm{H}), 3.62$ (q, $J=7.2 \mathrm{~Hz}, 1 \mathrm{H}), 1.48(\mathrm{~d}, J=7.2 \mathrm{~Hz}, 3 \mathrm{H}), 1.23$ (s, 9H); ${ }^{13}$ C NMR (100 MHz, $\left.\mathrm{CDCl}_{3}\right): \delta 172.2,161.3,148.7,137.3,122.3,122.1,50.9,49.9,28.7$, 
18.4; HRMS (EI): Calcd for $\mathrm{C}_{12} \mathrm{H}_{18} \mathrm{~N}_{2} \mathrm{O}$ : 206.1419; found: 206.1421 .

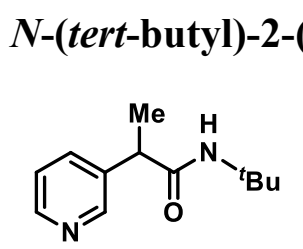

\section{(pyridin-3-yl)propanamide (3j)}

General procedure was followed on $0.5 \mathrm{mmol}$ scale with 1.5 equiv sodium tertbutoxide and 1.5 equiv tert-butyl isocyanide at $100^{\circ} \mathrm{C}$ for $10 \mathrm{~h}$. The reaction mixture was purification by flash column chromatography on silica gel (PE/EtOAc $=1 / 1-$ EtOAc) afforded $\mathbf{3 j}$ as a brown solid $(60.3 \mathrm{mg}, 58 \%) . \mathbf{R}_{\mathbf{f}}=0.30\left(\right.$ EtOAc); ${ }^{\mathbf{1}} \mathbf{H} \mathbf{~ N M R}$ $\left(400 \mathrm{MHz}, \mathrm{CDCl}_{3}\right): \delta 8.44-8.43(\mathrm{~m}, 2 \mathrm{H}), 7.67(\mathrm{dt}, J=8.0,2.0 \mathrm{~Hz}, 1 \mathrm{H}), 7.22(\mathrm{dd}, J=8.0,4.8 \mathrm{~Hz}, 1 \mathrm{H})$, 5.64 (bs, 1H), 3.42 (q, $J=7.2 \mathrm{~Hz}, 1 \mathrm{H}), 1.41$ (d, $J=7.2 \mathrm{~Hz}, 3 \mathrm{H}), 1.24$ (s, 9H); ${ }^{13} \mathbf{C}$ NMR (100 MHz, $\mathrm{CDCl}_{3}$ ): $\delta 172.4,148.9,148.3,137.8,134.9,123.8,51.4,45.0,28.7,19.1$; HRMS (EI): Calcd for $\mathrm{C}_{12} \mathrm{H}_{19} \mathrm{~N}_{2} \mathrm{O}[\mathrm{M}+\mathrm{H}]^{+}:$207.1492; found: 207.1492.

\section{$N$-(tert-butyl)-2-(pyridin-4-yl)propanamide (3k)}

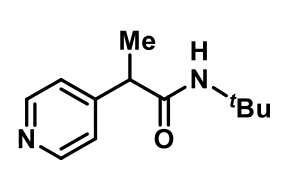

General procedure was followed on $0.5 \mathrm{mmol}$ scale with 1.5 equiv sodium tertbutoxide and 1.5 equiv tert-butyl isocyanide at $100{ }^{\circ} \mathrm{C}$ for $17 \mathrm{~h}$. The reaction mixture was purification by flash column chromatography on silica gel $(\mathrm{PE} / \mathrm{EtOAc}=1 / 1$ EtOAc) afforded 3k as a light yellow solid (73.6 mg, 71\%). $\mathbf{R}_{\mathbf{f}}=0.26\left(\right.$ EtOAc); ${ }^{1} \mathbf{H}$ NMR (400 MHz, $\left.\mathrm{CDCl}_{3}\right): \delta 8.44(\mathrm{~d}, J=1.2 \mathrm{~Hz}, 2 \mathrm{H}), 7.17(\mathrm{~d}, J=5.6 \mathrm{~Hz}, 2 \mathrm{H}), 5.74(\mathrm{bs}, 1 \mathrm{H}), 3.39$ (q, $J=7.2 \mathrm{~Hz}, 1 \mathrm{H}), 1.38(\mathrm{~d}, J=6.8 \mathrm{~Hz}, 3 \mathrm{H}), 1.22(\mathrm{~s}, 9 \mathrm{H}) ;{ }^{13} \mathbf{C} \mathbf{N M R}\left(100 \mathrm{MHz}, \mathrm{CDCl}_{3}\right): \delta 171.8,151.0$, 149.8, 122.7, 51.3, 46.9, 28.6, 18.4; HRMS (EI): Calcd for $\mathrm{C}_{12} \mathrm{H}_{19} \mathrm{~N}_{2} \mathrm{O} \quad[\mathrm{M}+\mathrm{H}]^{+}:$207.1492; found: 207.1491 .

\section{$N$-(tert-butyl)-2-(pyrazin-2-yl)propanamide (3I)}<smiles>[Y6]C(NC(=O)c1cnccn1)c1ccccc1</smiles>

General procedure was followed on $0.5 \mathrm{mmol}$ scale with 1.5 equiv sodium tertbutoxide and 1.5 equiv tert-butyl isocyanide at $100{ }^{\circ} \mathrm{C}$ for $13 \mathrm{~h}$. The reaction mixture was purification by flash column chromatography on silica gel $(\mathrm{PE} / \mathrm{EtOAc}=2 / 1)$ afforded $3 \mathbf{l}$ as a brown solid (70.1 $\mathrm{mg}, 68 \%)$. $\mathbf{R}_{\mathbf{f}}=0.36(\mathrm{PE} /$ EtOAc $=1 / 1) ;{ }^{1} \mathbf{H} \mathbf{~ N M R}$ (400 MHz, $\left.\mathrm{CDCl}_{3}\right): \delta 8.55(\mathrm{~s}, 1 \mathrm{H}), 8.44-8.43(\mathrm{~m}, 2 \mathrm{H}), 6.32(\mathrm{bs}, 1 \mathrm{H}), 3.63(\mathrm{q}, J=7.2 \mathrm{~Hz}, 1 \mathrm{H}), 1.51$ $(\mathrm{d}, J=7.2 \mathrm{~Hz}, 3 \mathrm{H}), 1.24(\mathrm{~s}, 9 \mathrm{H}) ;{ }^{13} \mathbf{C} \mathbf{N M R}\left(100 \mathrm{MHz}, \mathrm{CDCl}_{3}\right): \delta 171.1,156.5,144.1,143.6,143.2$, 51.2, 48.0, 28.7, 18.2; HRMS (EI): Calcd for $\mathrm{C}_{11} \mathrm{H}_{17} \mathrm{~N}_{3} \mathrm{O}$ : 207.1372; found: 207.1367.

\section{$N$-(tert-butyl)-2-phenyl-2-(pyridin-2-yl)acetamide (3m)}

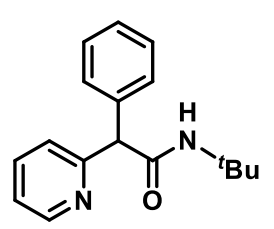

General procedure was followed on $0.5 \mathrm{mmol}$ scale with 1.5 equiv sodium tertbutoxide and 1.5 equiv tert-butyl isocyanide at $100{ }^{\circ} \mathrm{C}$ for $12 \mathrm{~h}$. The reaction mixture was purification by flash column chromatography on silica gel $(\mathrm{PE} / \mathrm{EtOAc}=5 / 1)$ afforded $\mathbf{3 m}$ as a brown solid (50.6 $\mathrm{mg}, 38 \%)$. $\mathbf{R}_{\mathbf{f}}=0.23(\mathrm{PE} / \mathrm{EtOAc}=3 / 1) ;{ }^{\mathbf{1}} \mathbf{H} \mathbf{~ N M R}$ $\left(400 \mathrm{MHz}, \mathrm{CDCl}_{3}\right): \delta 8.59(\mathrm{dd}, J=5.2,1.2 \mathrm{~Hz}, 1 \mathrm{H}), 7.66(\mathrm{td}, J=7.6,1.6 \mathrm{~Hz}, 1 \mathrm{H})$, 7.55 (bs, 1H), 7.42-7.40 (m, 2H), 7.34-7.29 (m, 3H), 7.27-7.23 (m, 1H), 7.20 (ddd, $J=7.6,4.8,1.2$ $\mathrm{Hz}, 1 \mathrm{H}), 4.94(\mathrm{~s}, 1 \mathrm{H}), 1.37$ (s, 9H); ${ }^{13} \mathbf{C}$ NMR (100 MHz, $\left.\mathrm{CDCl}_{3}\right): \delta 169.7,159.5,148.9,139.5,137.3$, 128.7, 128.2, 127.2, 124.5, 122.2, 61.6, 51.2, 28.7; HRMS (ESI): Calcd for $\mathrm{C}_{17} \mathrm{H}_{21} \mathrm{~N}_{2} \mathrm{O}[\mathrm{M}+\mathrm{H}]^{+}$: 269.1654; found: 269.1653 . 


\section{$N$-(tert-butyl)-2,2-diphenylacetamide (3n)}<smiles></smiles>

General procedure was followed on $0.2 \mathrm{mmol}$ scale with 1.1 equiv sodium tertbutoxide and 1.1 equiv tert-butyl isocyanide at $100{ }^{\circ} \mathrm{C}$ for $17.5 \mathrm{~h}$. The reaction mixture was purification by flash column chromatography on silica gel (PE/EtOAc $=30 / 1-10 / 1)$ afforded 3nas a light yellow solid $(26.8 \mathrm{mg}, 50 \%) . \mathbf{R}_{\mathbf{f}}=0.28$

$(\mathrm{PE} / \mathrm{EtOAc}=10 / 1) ;{ }^{1} \mathbf{H} \mathbf{~ N M R}\left(400 \mathrm{MHz}, \mathrm{CDCl}_{3}\right): \delta$ 7.41-7.39 (m, 1H), 7.37-7.32 (m, 5H), 7.29-7.26 (m, 4H), 5.44 (bs, $1 \mathrm{H}), 4.83(\mathrm{~s}, 1 \mathrm{H}), 1.34$ (s, 9H); ${ }^{13} \mathbf{C}$ NMR $\left(100 \mathrm{MHz}, \mathrm{CDCl}_{3}\right): \delta$ $171.2,144.0,140.0,128.9,128.8,128.6,127.6,127.2,126.7,59.9,51.6,28.8$. The spectral data is consistent with previously reported literature values. ${ }^{[15]}$

\section{$N$-(tert-butyl)-2-(naphthalen-2-yl)-2-phenylacetamide (3o)}

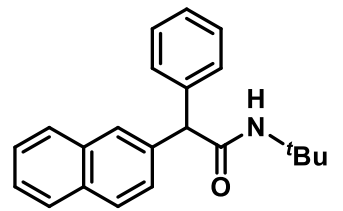

General procedure was followed on $0.5 \mathrm{mmol}$ scale with 2.0 equiv sodium tertbutoxide and 1.5 equiv tert-butyl isocyanide at $100^{\circ} \mathrm{C}$ for $10 \mathrm{~h}$ and then $120^{\circ} \mathrm{C}$ $5 \mathrm{~h}$. The reaction mixture was purification by flash column chromatography on silica gel $(\mathrm{PE} / \mathrm{EtOAc}=20 / 1-5 / 1)$ afforded 30 as a light yellow solid $(63.5 \mathrm{mg}$, $40 \%) . \mathbf{R}_{\mathbf{f}}=0.26(\mathrm{PE} / \mathrm{EtOAc}=10 / 1) ;{ }^{1} \mathbf{H} \mathbf{~ N M R}\left(400 \mathrm{MHz}, \mathrm{CDCl}_{3}\right): \delta 7.83-7.78(\mathrm{~m}, 3 \mathrm{H}), 7.72(\mathrm{~s}, 1 \mathrm{H})$, 7.49-7.45 (m, 2H), 7.40 (dd, $J=8.4,1.2 \mathrm{~Hz}, 1 \mathrm{H}), 7.37-7.26(\mathrm{~m}, 5 \mathrm{H}), 5.49$ (bs, $1 \mathrm{H}), 4.99(\mathrm{~s}, 1 \mathrm{H}), 1.36$ (s, 9H); ${ }^{13} \mathbf{C}$ NMR $\left(100 \mathrm{MHz}, \mathrm{CDCl}_{3}\right): \delta 171.1,139.9,137.5,133.6,132.6,129.1,128.9,128.5,128.1$, 127.7, 127.6, 127.3, 127.2, 126.3, 126.1, 60.1, 51.8, 28.8; HRMS (EI): Calcd for $\mathrm{C}_{22} \mathrm{H}_{23} \mathrm{NO}$ : 317.1780; found: 317.1774 .

\section{$N$-((3s,5s,7s)-adamantan-1-yl)-2-phenylpropanamide (3p)}

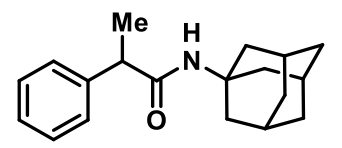
General procedure was followed on $0.5 \mathrm{mmol}$ scale with 2.0 equiv sodium tertbutoxide and 1.5 equiv 1 -adamantyl isocyanide at $100{ }^{\circ} \mathrm{C}$ for $11.5 \mathrm{~h}$. The reaction was quenched with $3 \mathrm{M}$ aq. $\mathrm{HCl}$ for $1 \mathrm{~h}$. The crude product was purification by flash column chromatography on silica gel $(\mathrm{PE} / \mathrm{EtOAc}=20 / 1-10 / 1)$ afforded $\mathbf{3 p}$ as a white solid $(128.0$ $\mathrm{mg}, 45 \%) . \mathbf{R}_{\mathbf{f}}=0.25(\mathrm{PE} / \mathrm{EtOAc}=20 / 1) ;{ }^{1} \mathbf{H}$ NMR $\left(400 \mathrm{MHz}, \mathrm{CDCl}_{3}\right): \delta 7.36-7.24(\mathrm{~m}, 5 \mathrm{H}), 5.07$ (bs, 1H), 3.47 (q, $J=7.2 \mathrm{~Hz}, 1 \mathrm{H}$ ), 2.04 (br s, 3H), 1.92 (d, $J=3.2 \mathrm{~Hz}, 6 \mathrm{H}$ ), 1.64 (t, $J=2.8 \mathrm{~Hz}, 6 \mathrm{H}$ ), 1.48 $(\mathrm{d}, J=7.2 \mathrm{~Hz}, 3 \mathrm{H}) ;{ }^{13} \mathbf{C}$ NMR $\left(100 \mathrm{MHz}, \mathrm{CDCl}_{3}\right): \delta 173.4,142.1,128.9,127.6,127.1,51.9,47.9$, $41.5,36.4,29.5,18.8$. The spectral data is consistent with previously reported literature values. ${ }^{[16]}$ 
Table S1. Other attempted substrates ${ }^{a}$

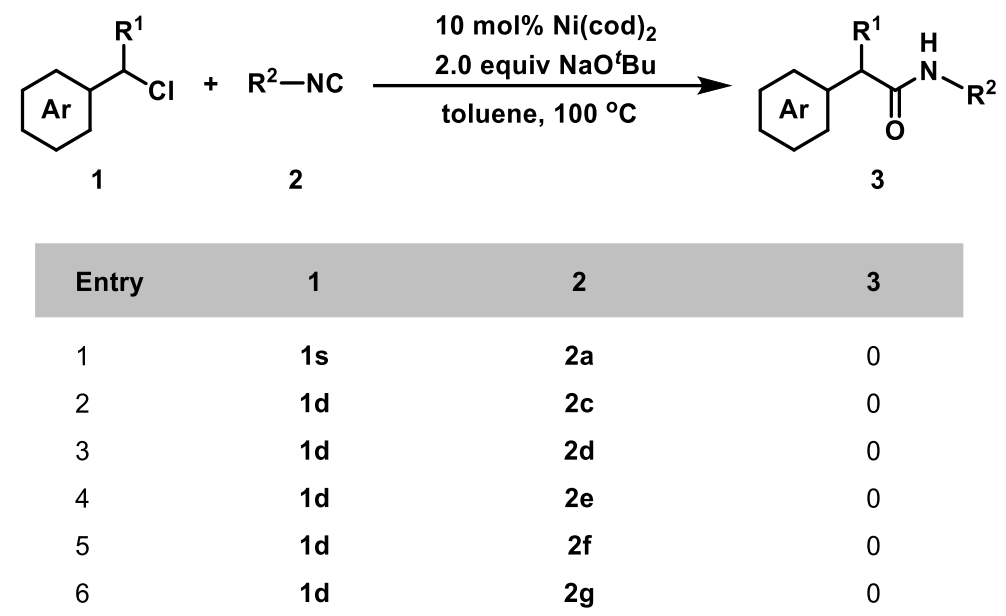

${ }^{a}$ Reaction conditions: secondary benzyl chloride 1 (1.0 equiv), isocyanide 2 (1.5 equiv), $\mathrm{Ni}(\mathrm{cod})_{2}(10 \mathrm{~mol} \%), \mathrm{NaO}^{t} \mathrm{Bu}\left(2.0\right.$ equiv), toluene $(0.1 \mathrm{M}), 100^{\circ} \mathrm{C}$, $12 \mathrm{~h}$. Then $1 \mathrm{M} \mathrm{HCl}$, r.t., $5 \mathrm{~min}$.

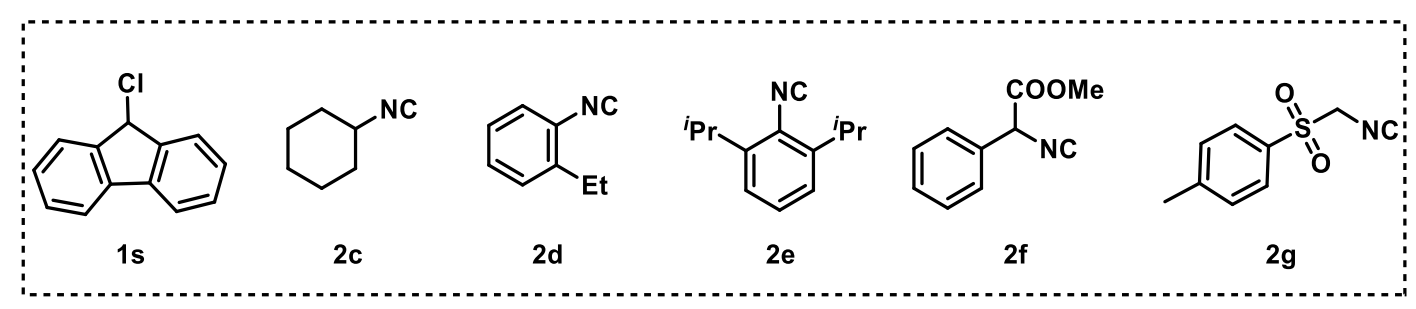

The desired amides could not be detected when $\mathbf{1 s}, \mathbf{2 c}, \mathbf{2 d}, \mathbf{2 e}, \mathbf{2 f}, \mathbf{2 g}$ were employed as substrates. 


\section{Experiment of Capturing Intermediate}

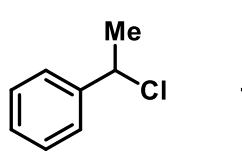

$1 d$

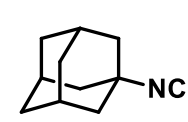

2b

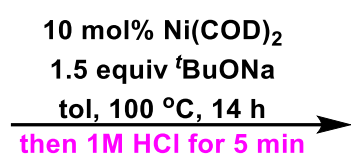

1.5 equiv<smiles>CC(C(=O)NC12CC3CC(CC(C3)C1)C2)c1ccccc1</smiles>

$3 p, 102 \%$<smiles>CC(=NC12CC3CC(CC(C3)C1)C2)c1ccccc1</smiles>

D, $48 \%$

In air, sodium tert-butoxide ( 1.5 equiv, $0.75 \mathrm{mmol}, 72.1 \mathrm{mg}$ ) and isocyanide $\mathbf{2 b}$ (1.5 equiv, 0.75 mmol, $120.9 \mathrm{mg}$ ) were added to a Schlenk tube equipped with a stir bar. T The vessel was moved to the glovebox, $\mathrm{Ni}(\mathrm{COD})_{2}(10 \mathrm{~mol} \%, 0.05 \mathrm{mmol}, 13.8 \mathrm{mg})$ was added. Then, the vessel was taken out of the glovebox before toluene $(0.1 \mathrm{M}, 5 \mathrm{~mL})$ and benzyl chloride $\mathbf{1 d}(1.0$ equiv, $0.2 \mathrm{mmol}, 36 \mu \mathrm{L}$ ) were added in turn by syringe. The reaction mixture was stirred at $100{ }^{\circ} \mathrm{C}$ oil bath for $14 \mathrm{~h}$ until complete by TLC analysis. When the starting material was consumed, the reaction was quenched with $1 \mathrm{M}$ aq. $\mathrm{HCl}$ for 5 minutes. The reaction mixture was monitored by GC-MS using dodecane as internal standard. It was found the desired amide was observed in $102 \%$ uncorrected GC yield, as well as ketenimine intermediate $\mathbf{D}$ in $48 \%$ uncorrected GC yield, which was confirmed by MS detector. This experiment proved that the amide product was formed via hydrolysis of ketenimine intermediate.
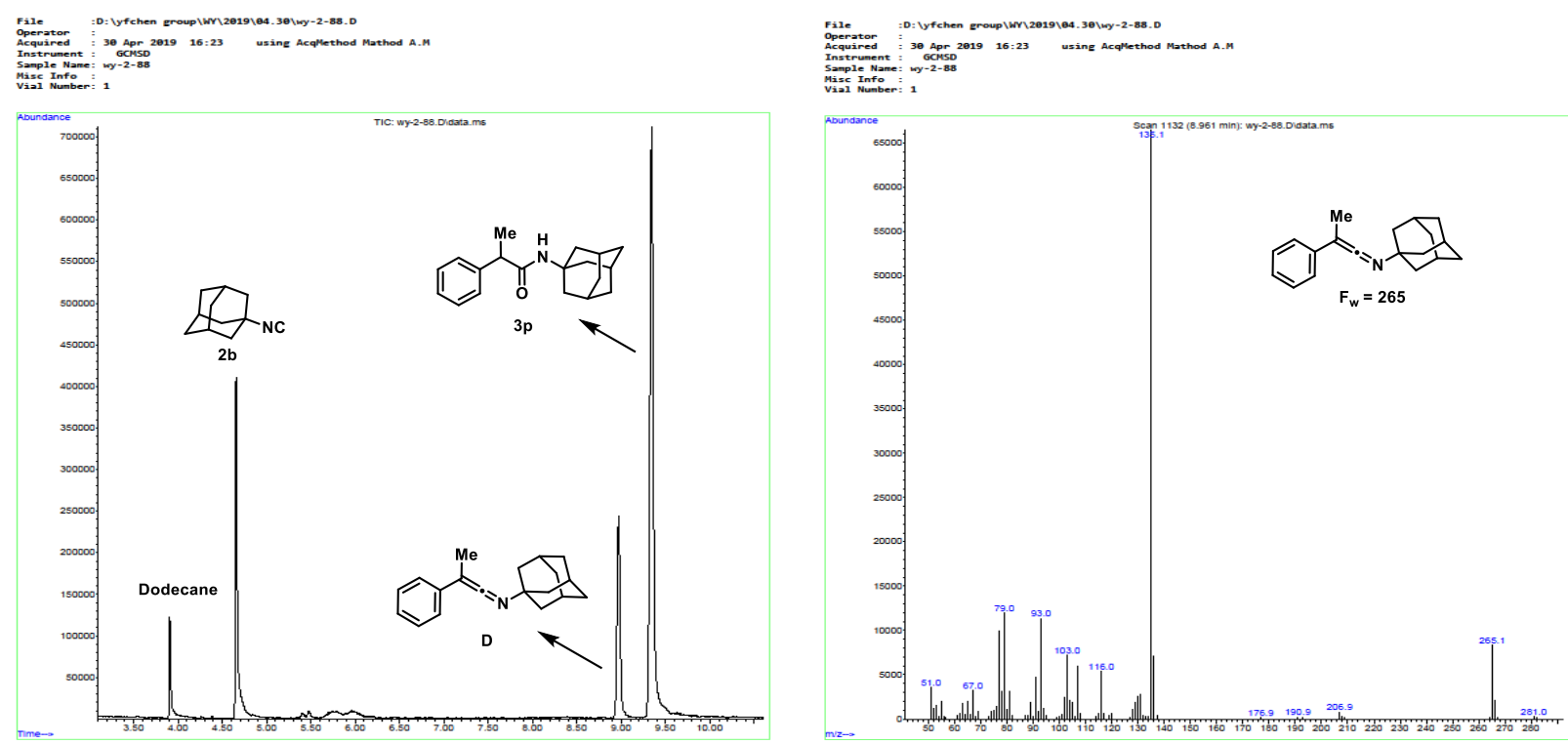
NMR Spectra

${ }^{1} \mathrm{H}$ NMR-spectrum(400 $\left.\mathrm{MHz}, \mathrm{CDCl}_{3}\right)$ of $1 \mathrm{c}$

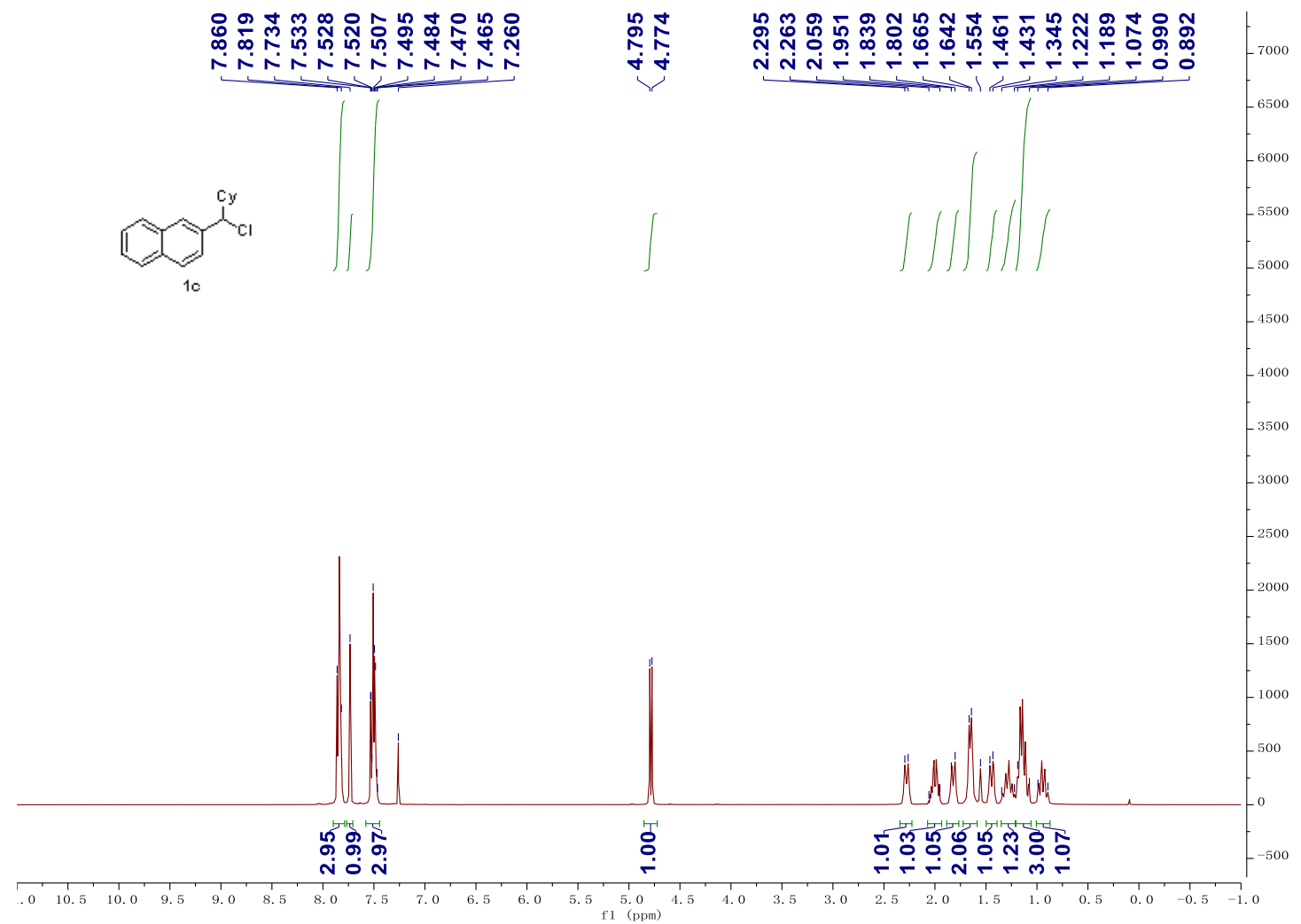

${ }^{13} \mathrm{C}$ NMR-spectrum(100 MHz, $\left.\mathrm{CDCl}_{3}\right)$ of $1 \mathrm{c}$

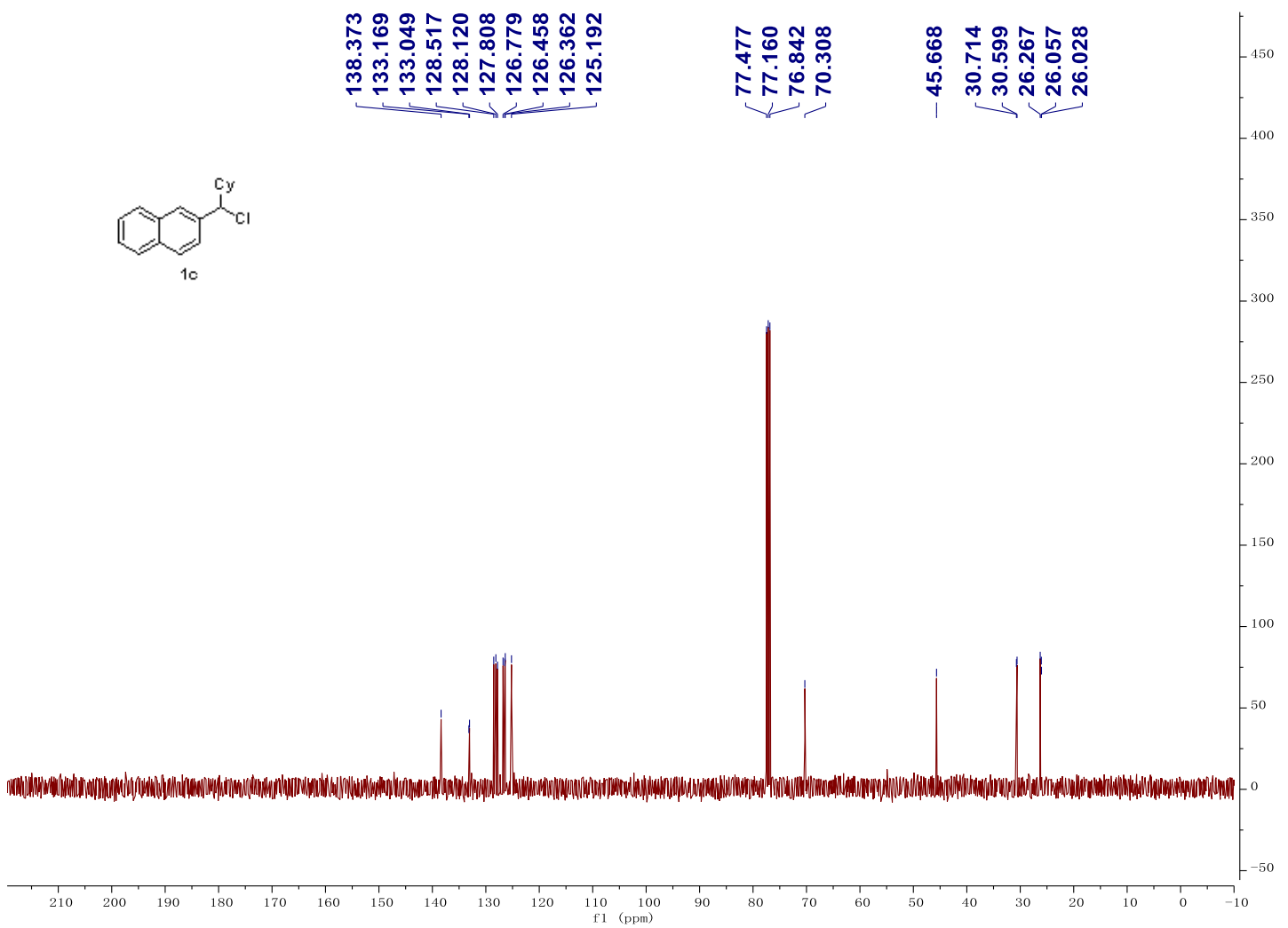


${ }^{1} \mathrm{H}$ NMR-spectrum(400 $\left.\mathrm{MHz}, \mathrm{CDCl}_{3}\right)$ of $1 \mathrm{l}$

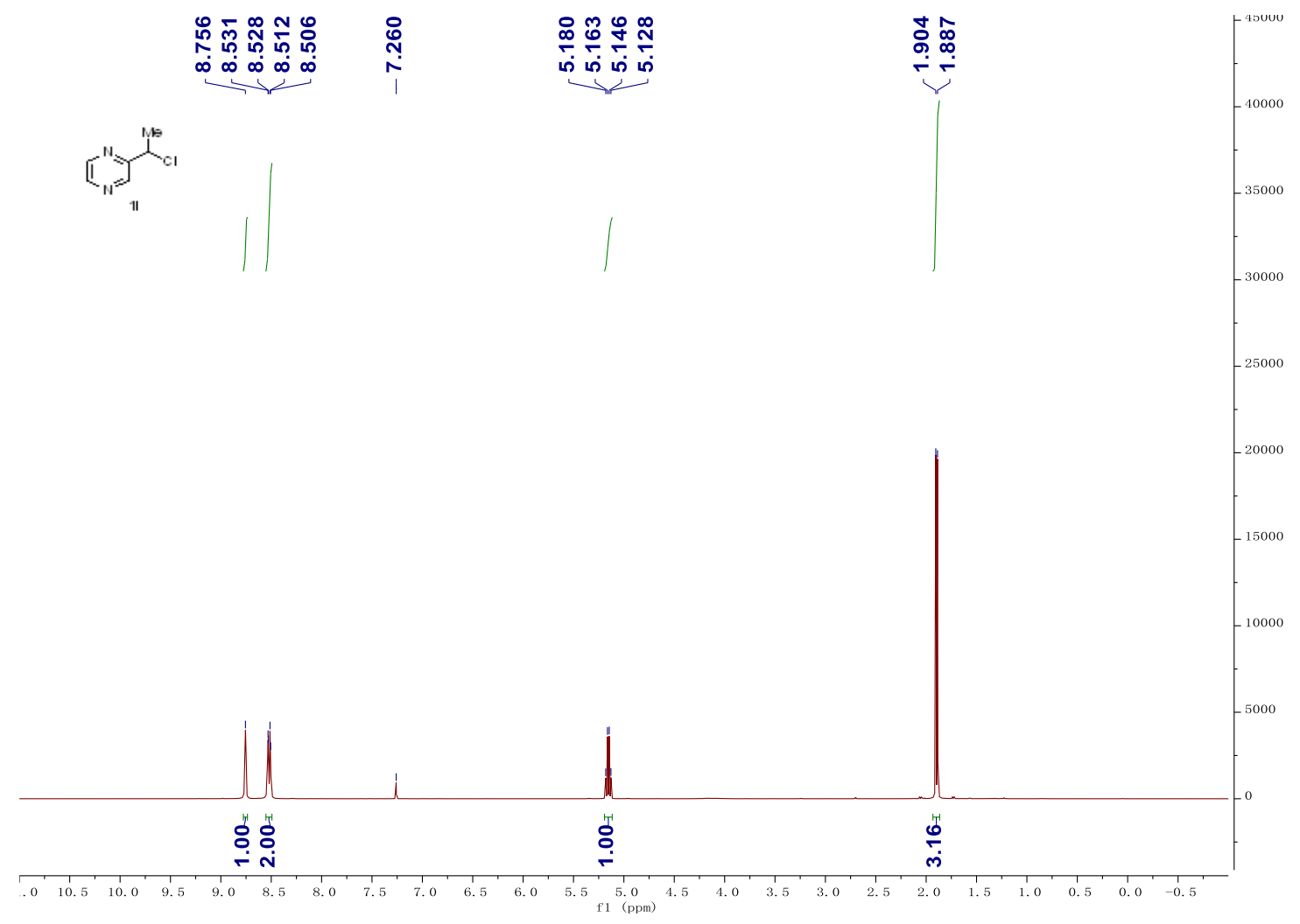

${ }^{13} \mathrm{C}$ NMR-spectrum(100 MHz, $\left.\mathrm{CDCl}_{3}\right)$ of 11

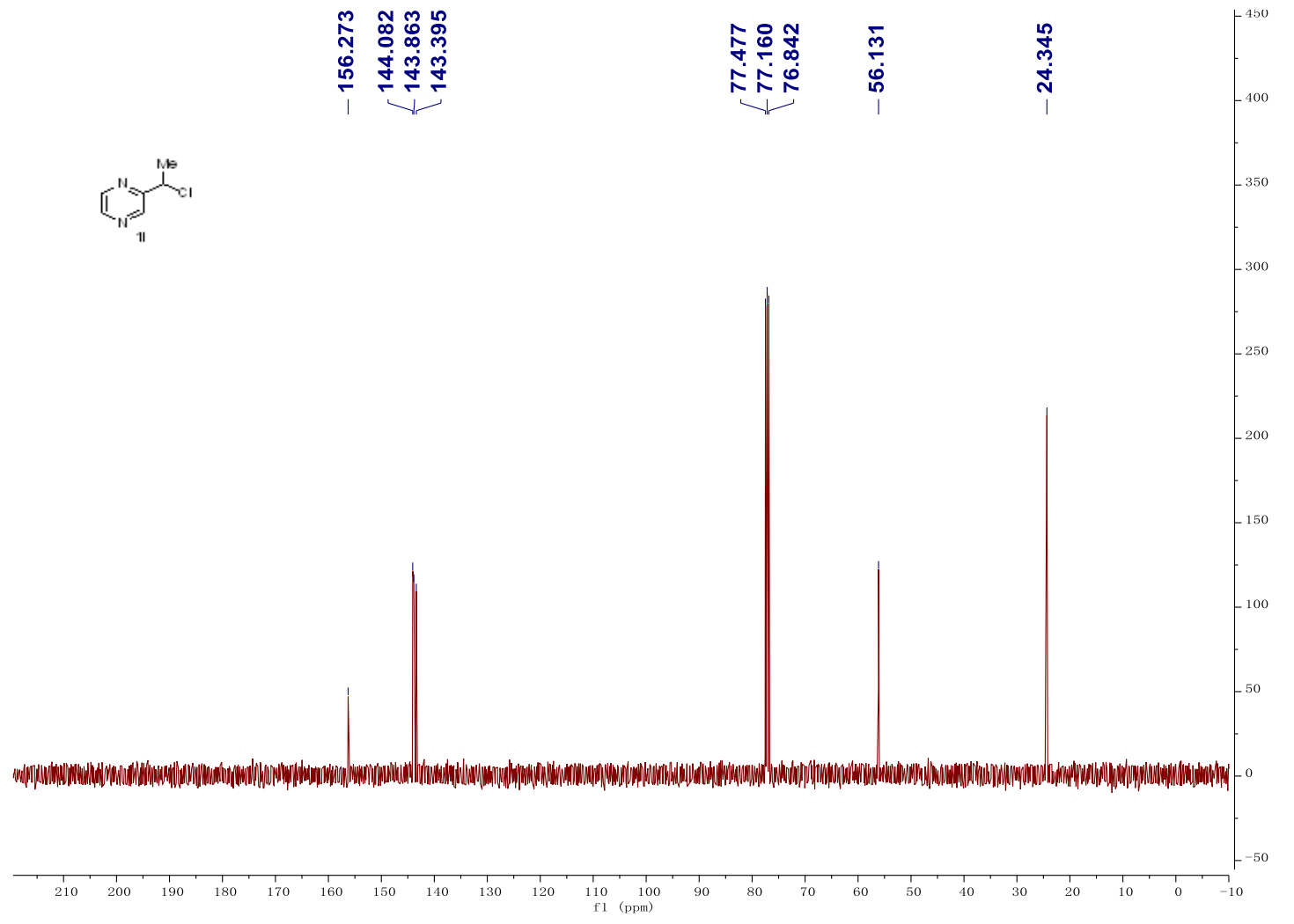


${ }^{1} \mathrm{H}$ NMR-spectrum(400 $\left.\mathrm{MHz}, \mathrm{CDCl}_{3}\right)$ of $3 \mathrm{a}$

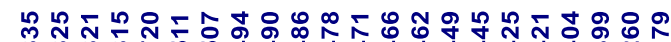

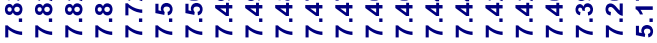

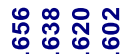

$\dot{m} \dot{m} \dot{m}$

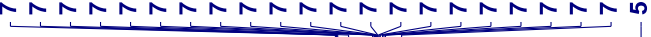

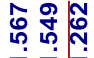

2

3
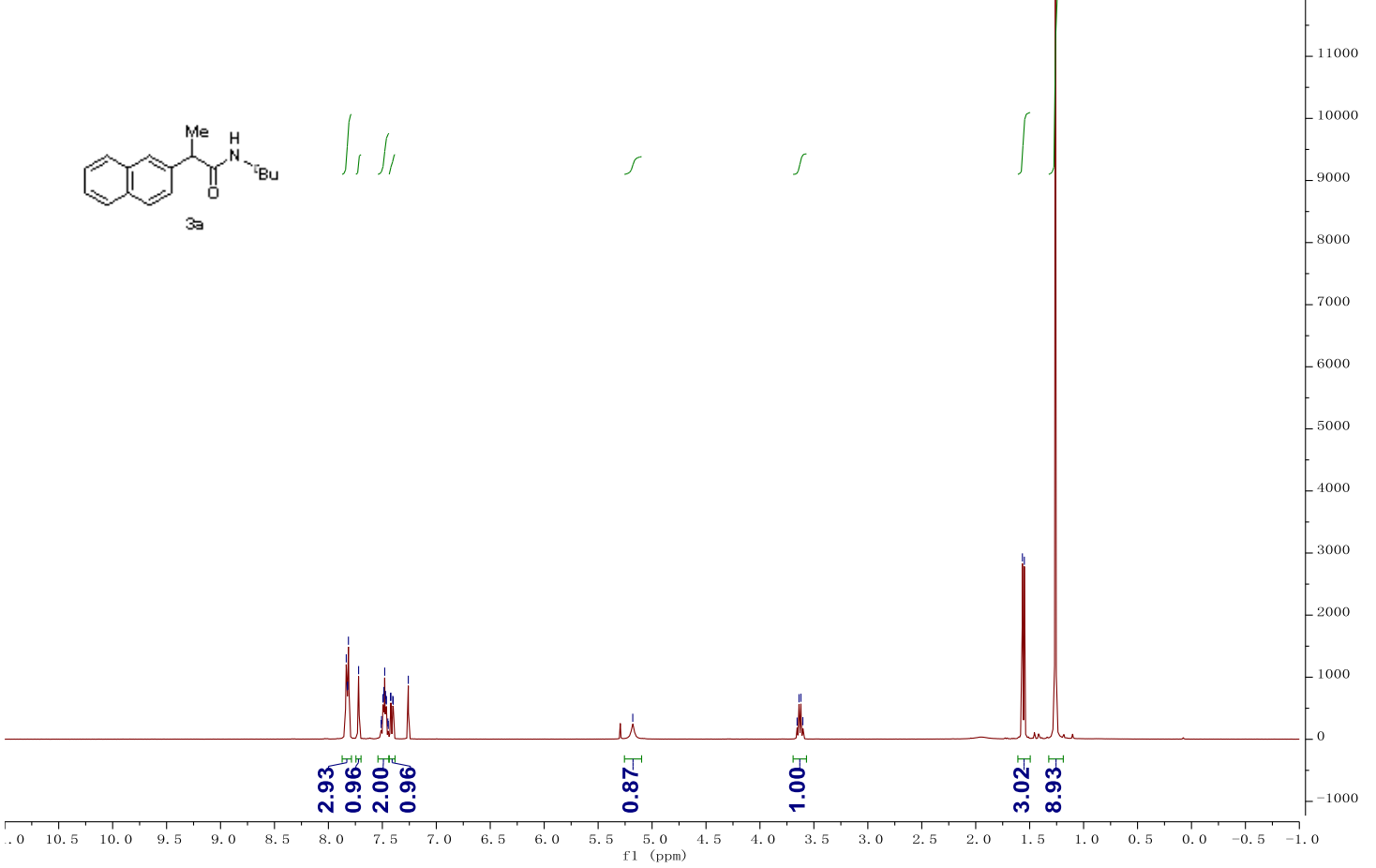

${ }^{13} \mathrm{C}$ NMR-spectrum(100 $\left.\mathrm{MHz}, \mathrm{CDCl}_{3}\right)$ of 3a

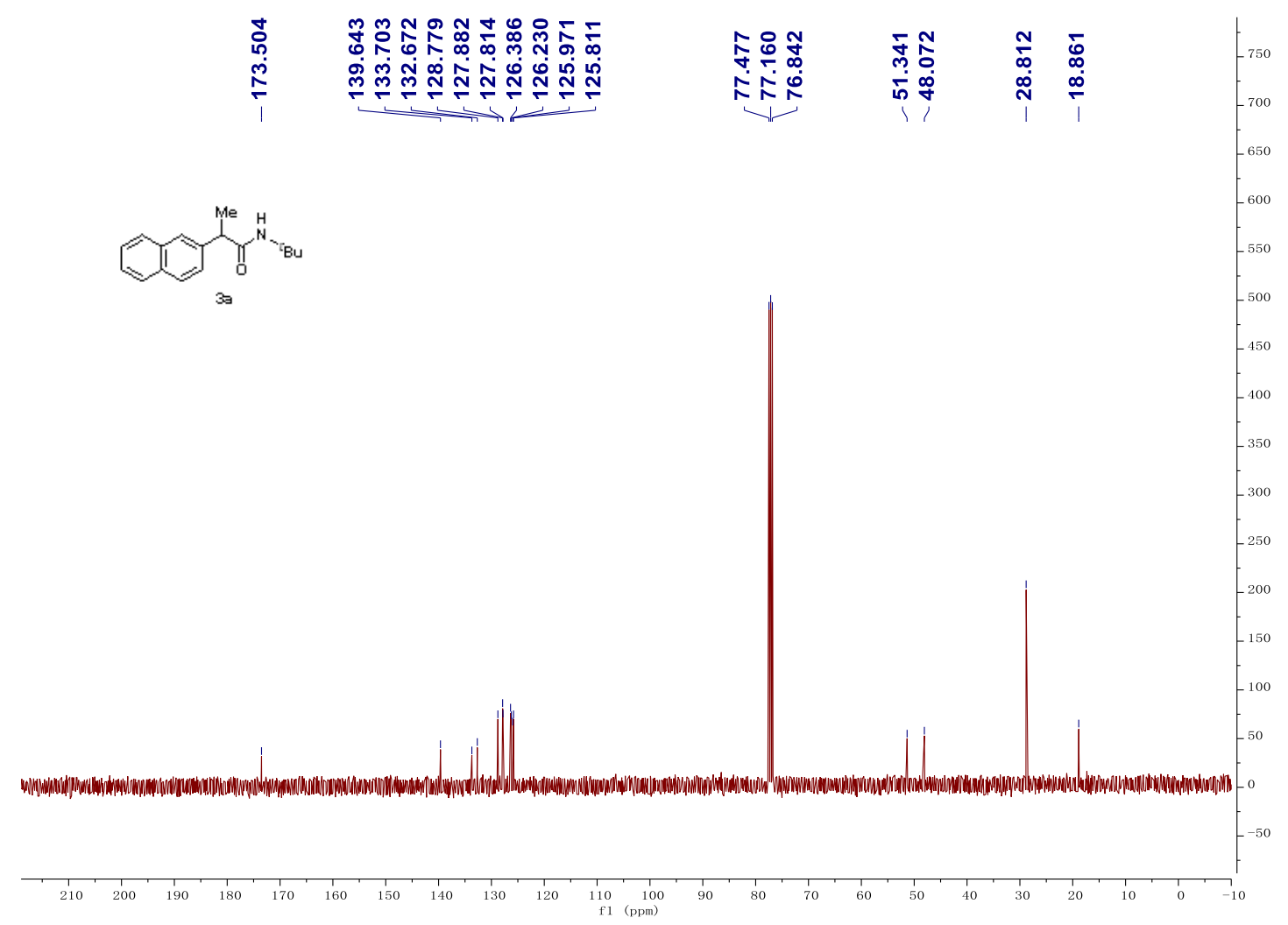


${ }^{1} \mathrm{H}$ NMR-spectrum(400 $\left.\mathrm{MHz}, \mathrm{CDCl}_{3}\right)$ of $3 \mathrm{~b}$

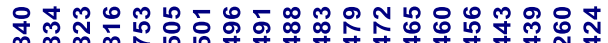

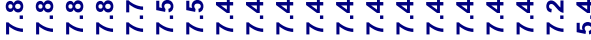

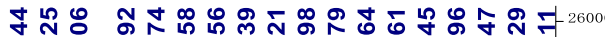

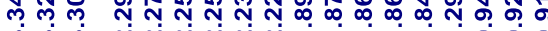

mต

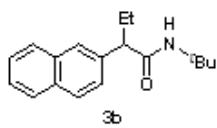

3

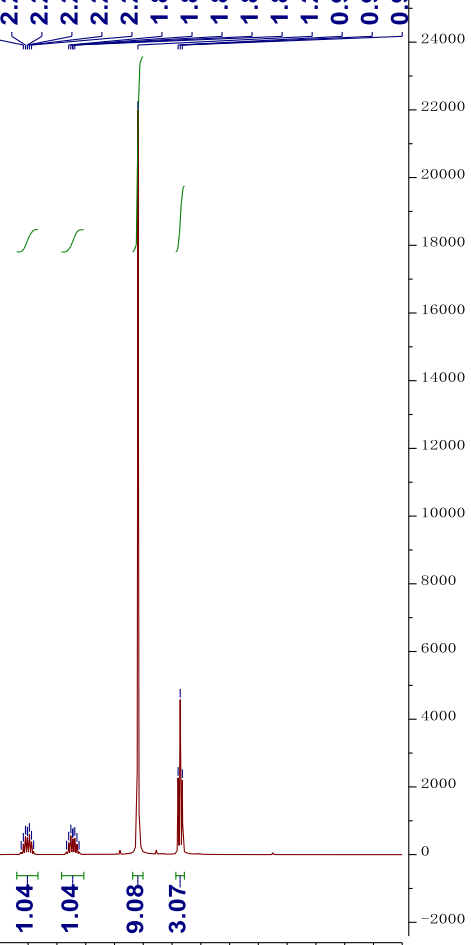

${ }^{13} \mathrm{C}$ NMR-spectrum(100 $\left.\mathrm{MHz}, \mathrm{CDCl}_{3}\right)$ of $3 \mathrm{~b}$

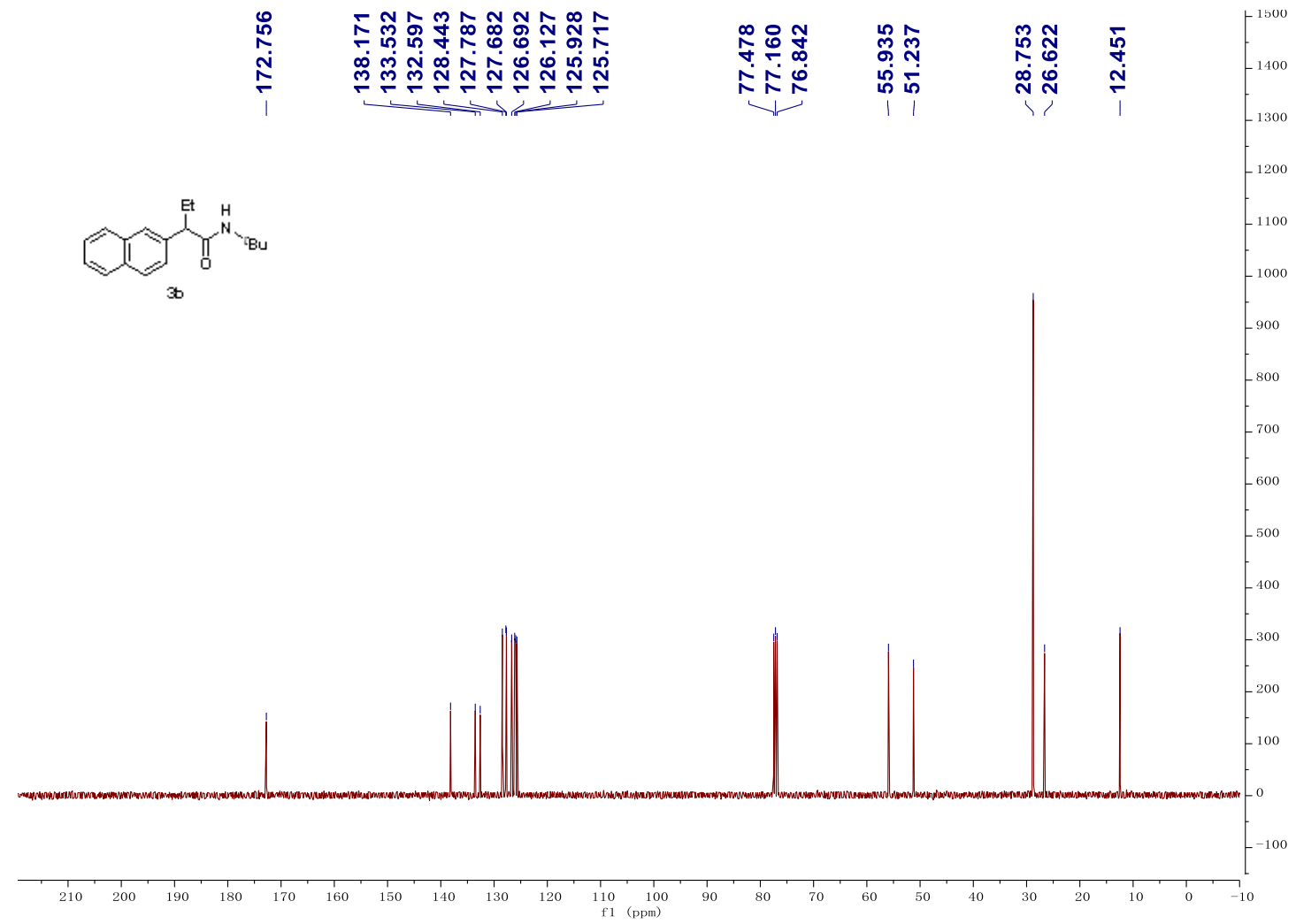


${ }^{1} \mathrm{H}$ NMR-spectrum(400 $\left.\mathrm{MHz}, \mathrm{CDCl}_{3}\right)$ of $3 \mathrm{c}$

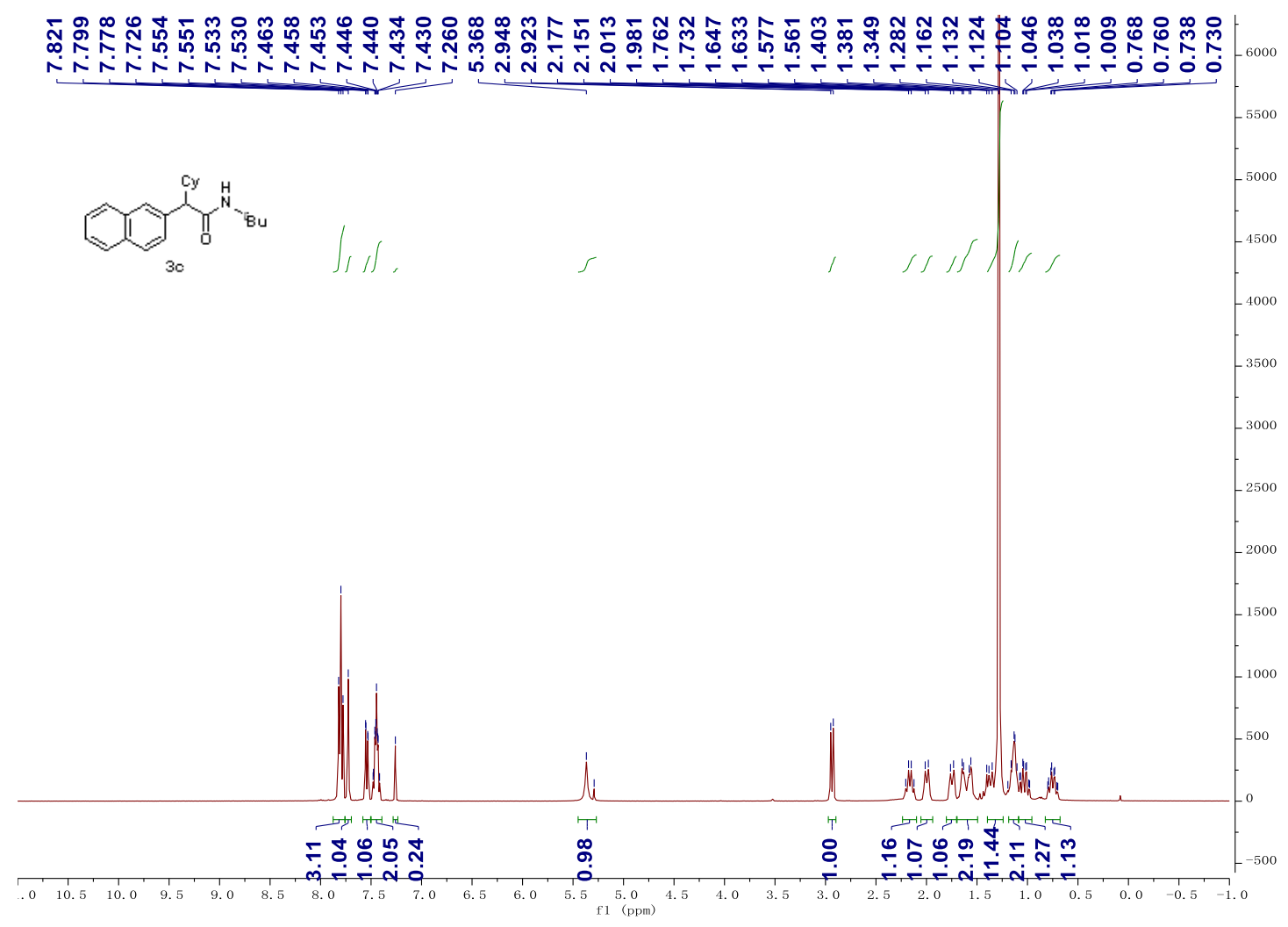

${ }^{13} \mathrm{C}$ NMR-spectrum(100 MHz, $\left.\mathrm{CDCl}_{3}\right)$ of $3 \mathrm{c}$
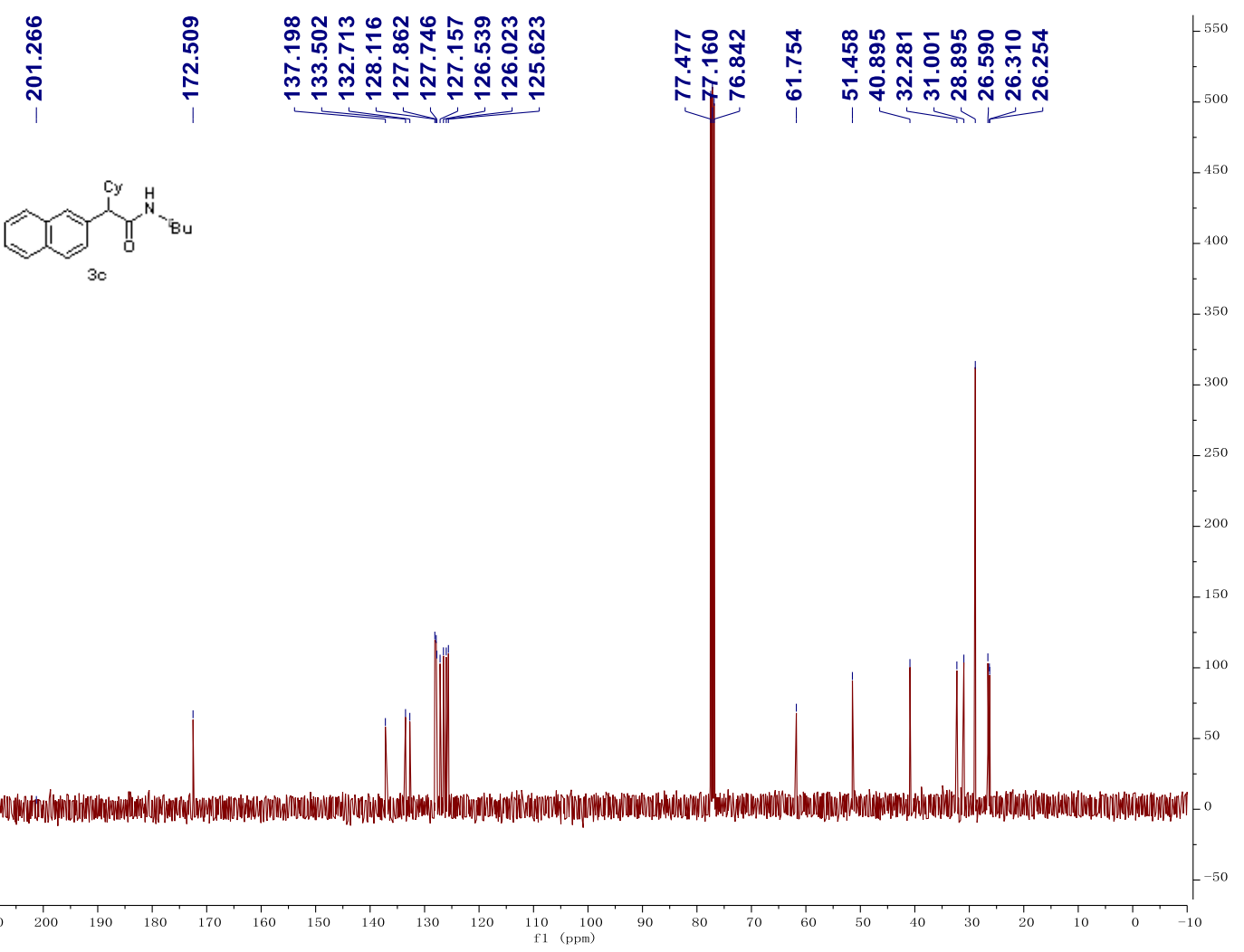
${ }^{1} \mathrm{H}$ NMR-spectrum(400 $\left.\mathrm{MHz}, \mathrm{CDCl}_{3}\right)$ of 3d

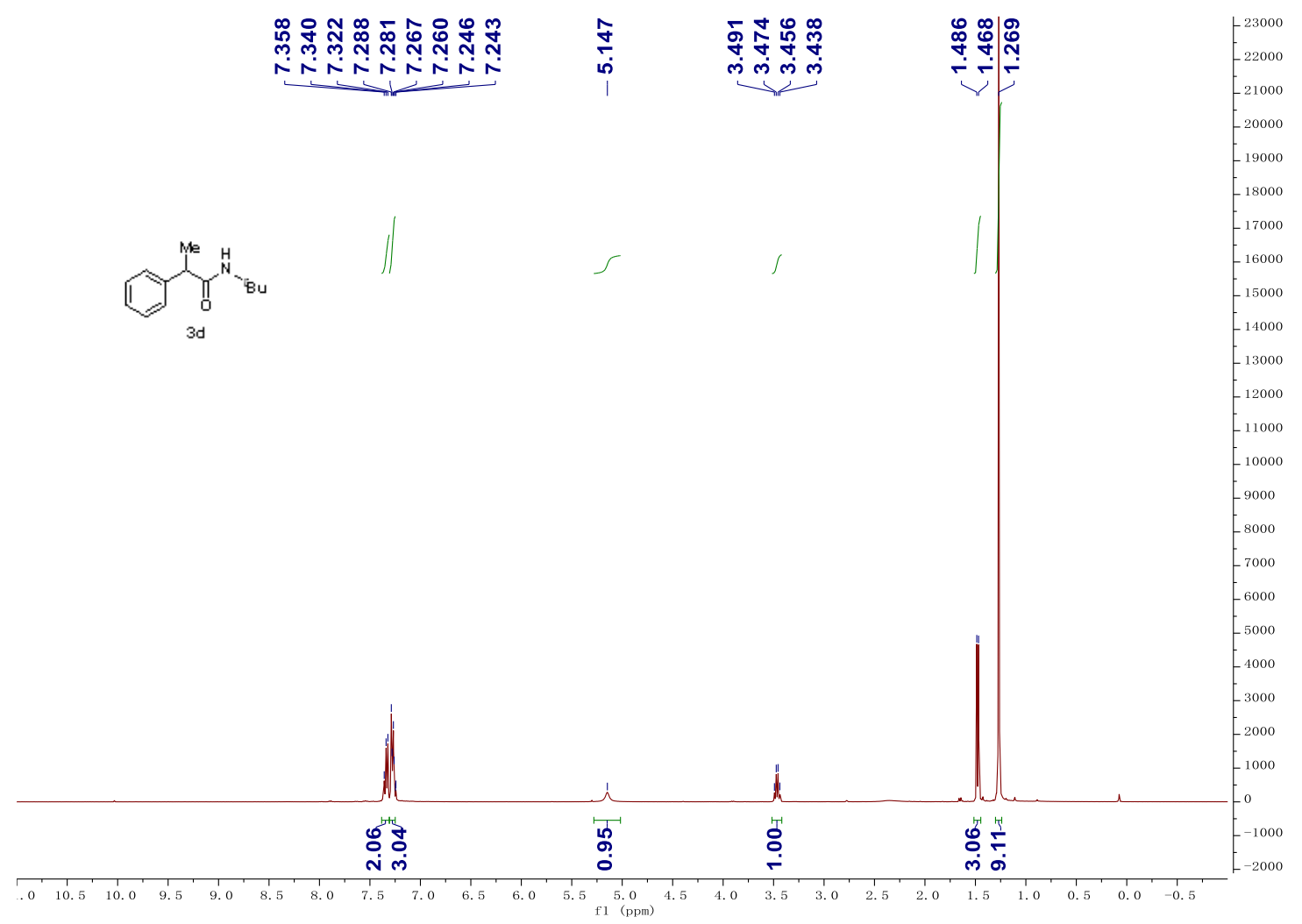

${ }^{13} \mathrm{C}$ NMR-spectrum(100 MHz, $\left.\mathrm{CDCl}_{3}\right)$ of $3 \mathrm{~d}$

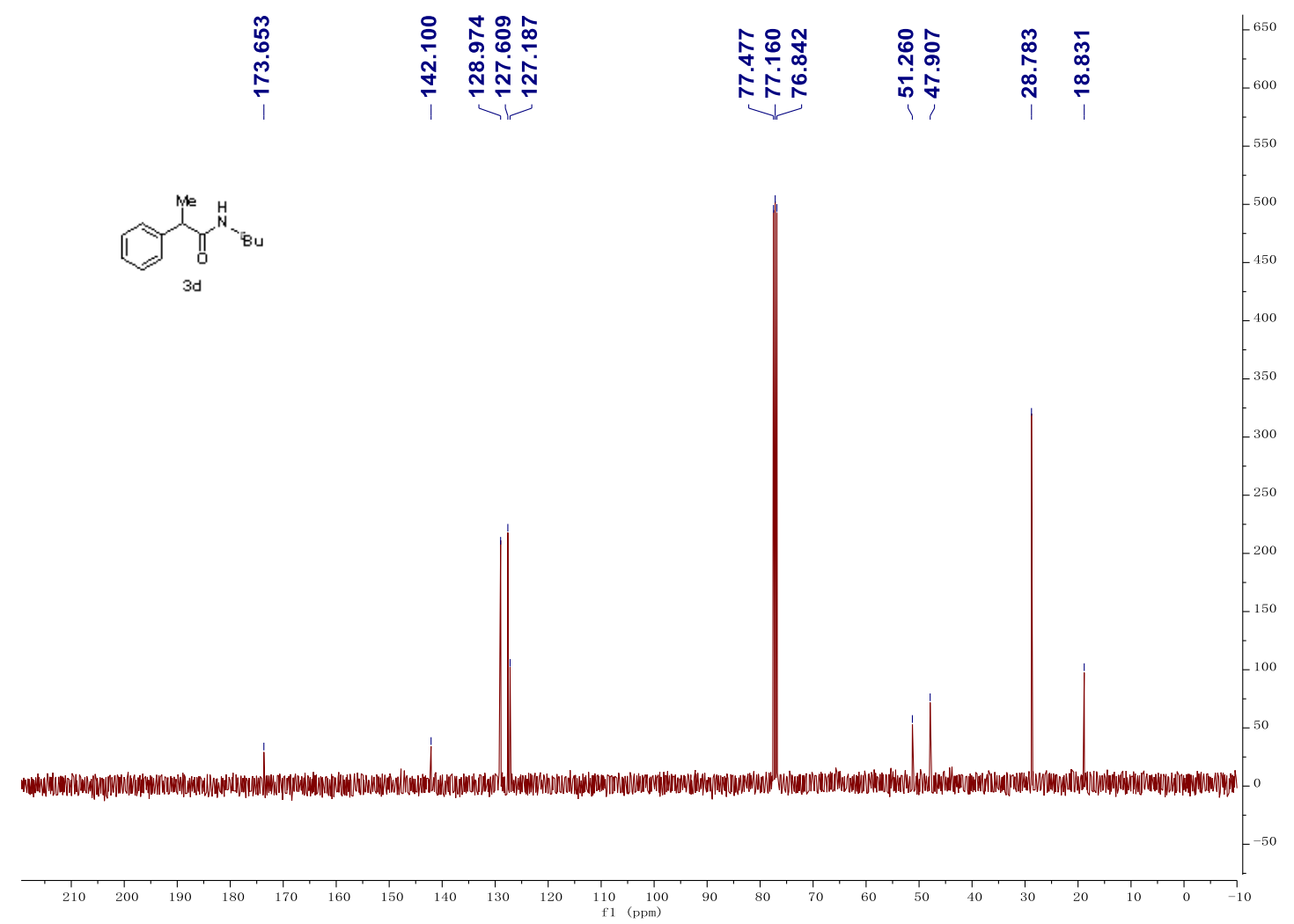


${ }^{1} \mathrm{H}$ NMR-spectrum(400 $\left.\mathrm{MHz}, \mathrm{CDCl}_{3}\right)$ of $3 \mathrm{e}$

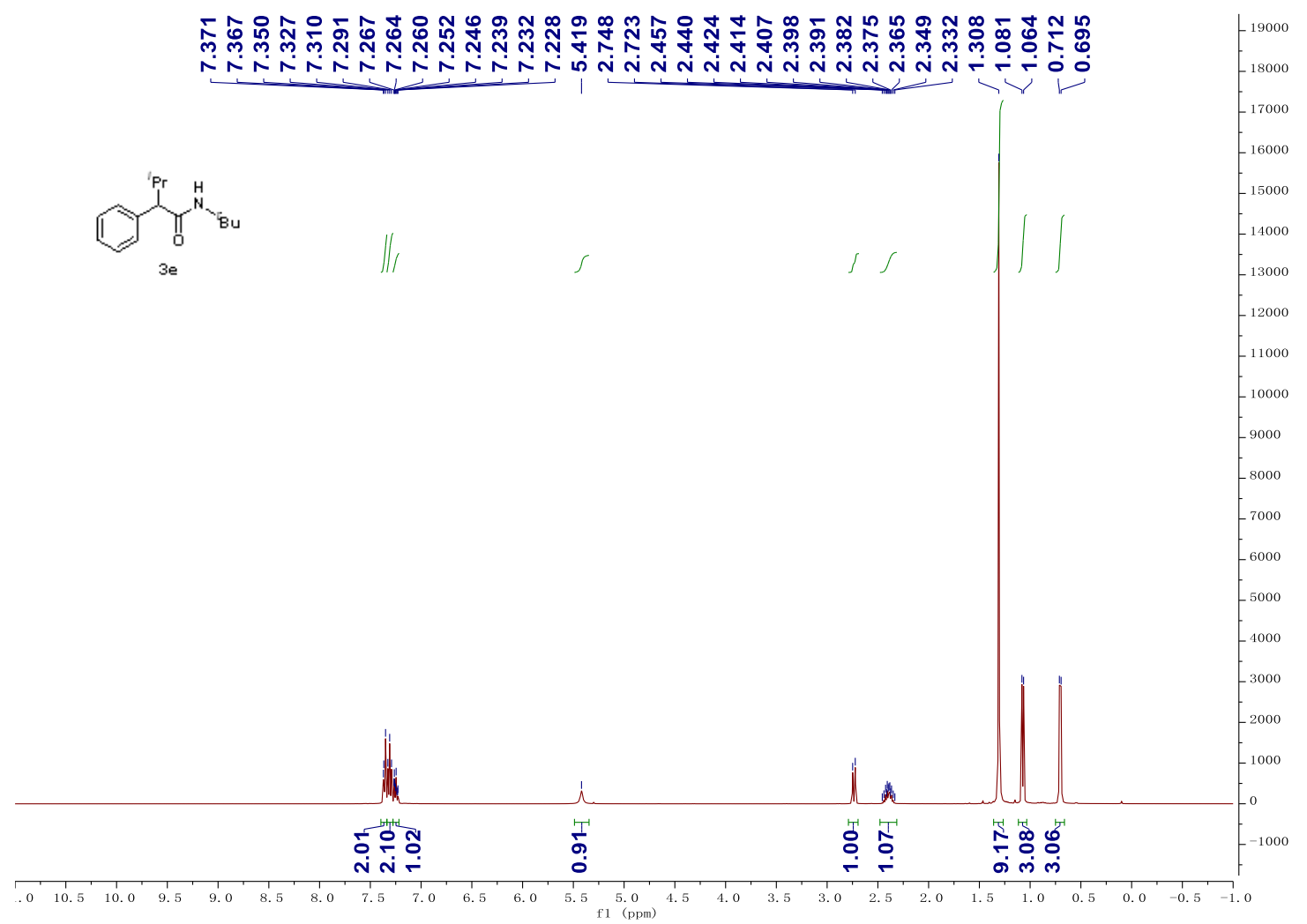

${ }^{13} \mathrm{C}$ NMR-spectrum(100 $\left.\mathrm{MHz}, \mathrm{CDCl}_{3}\right)$ of $3 \mathrm{e}$

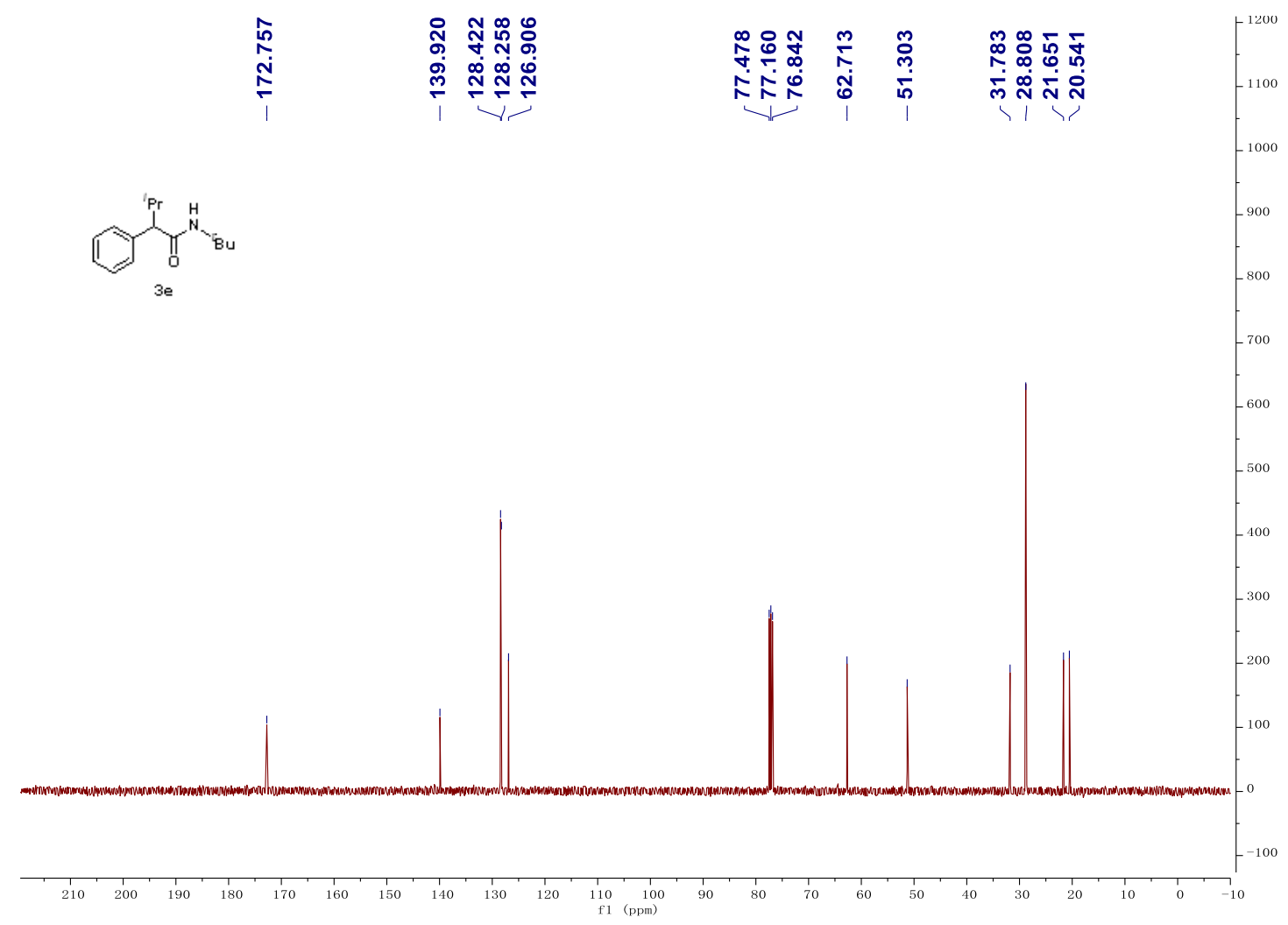


${ }^{1} \mathrm{H}$ NMR-spectrum(400 $\left.\mathrm{MHz}, \mathrm{CDCl}_{3}\right)$ of $3 \mathrm{f}$

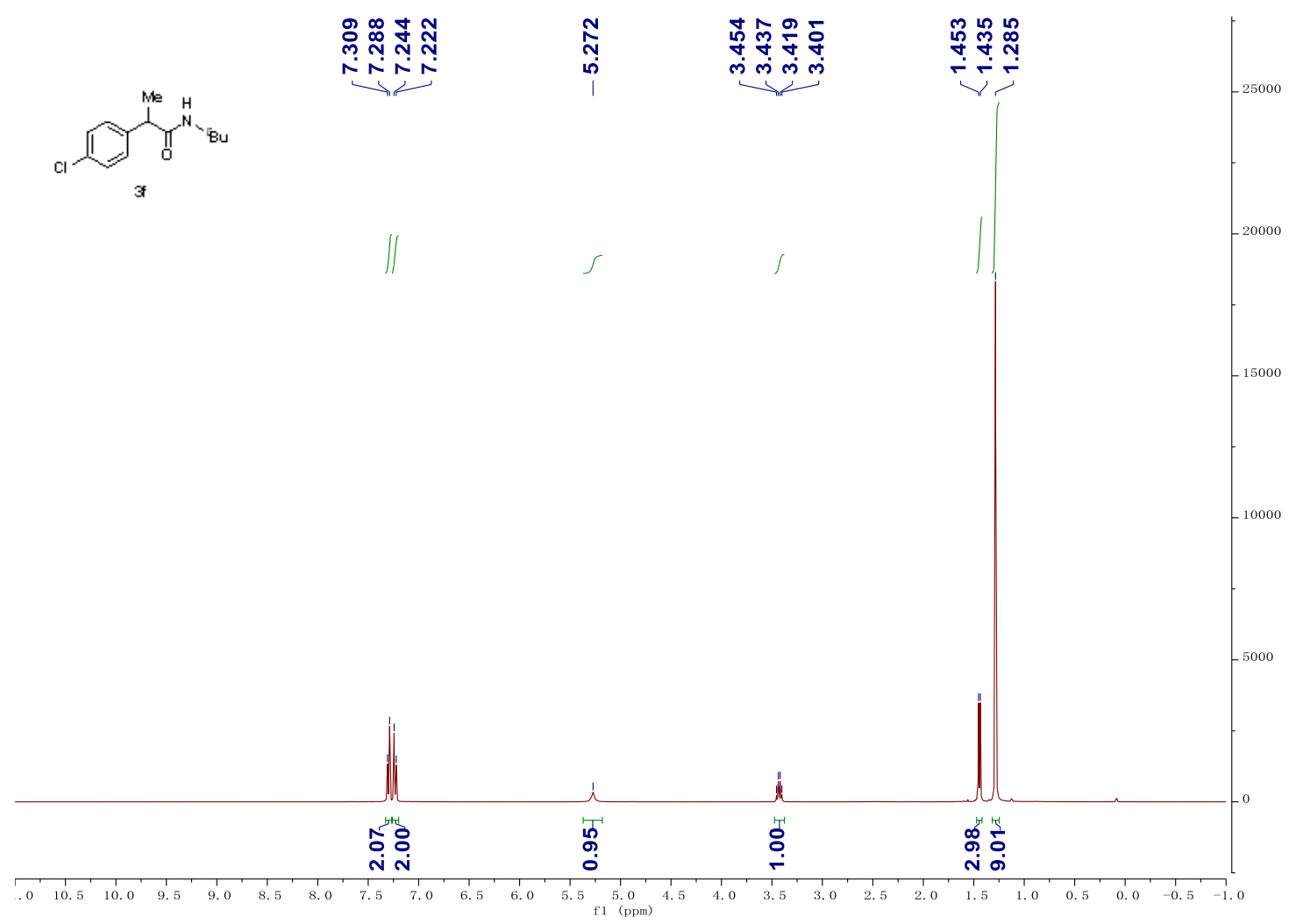

${ }^{13} \mathrm{C}$ NMR-spectrum(100 MHz, $\left.\mathrm{CDCl}_{3}\right)$ of $3 f$

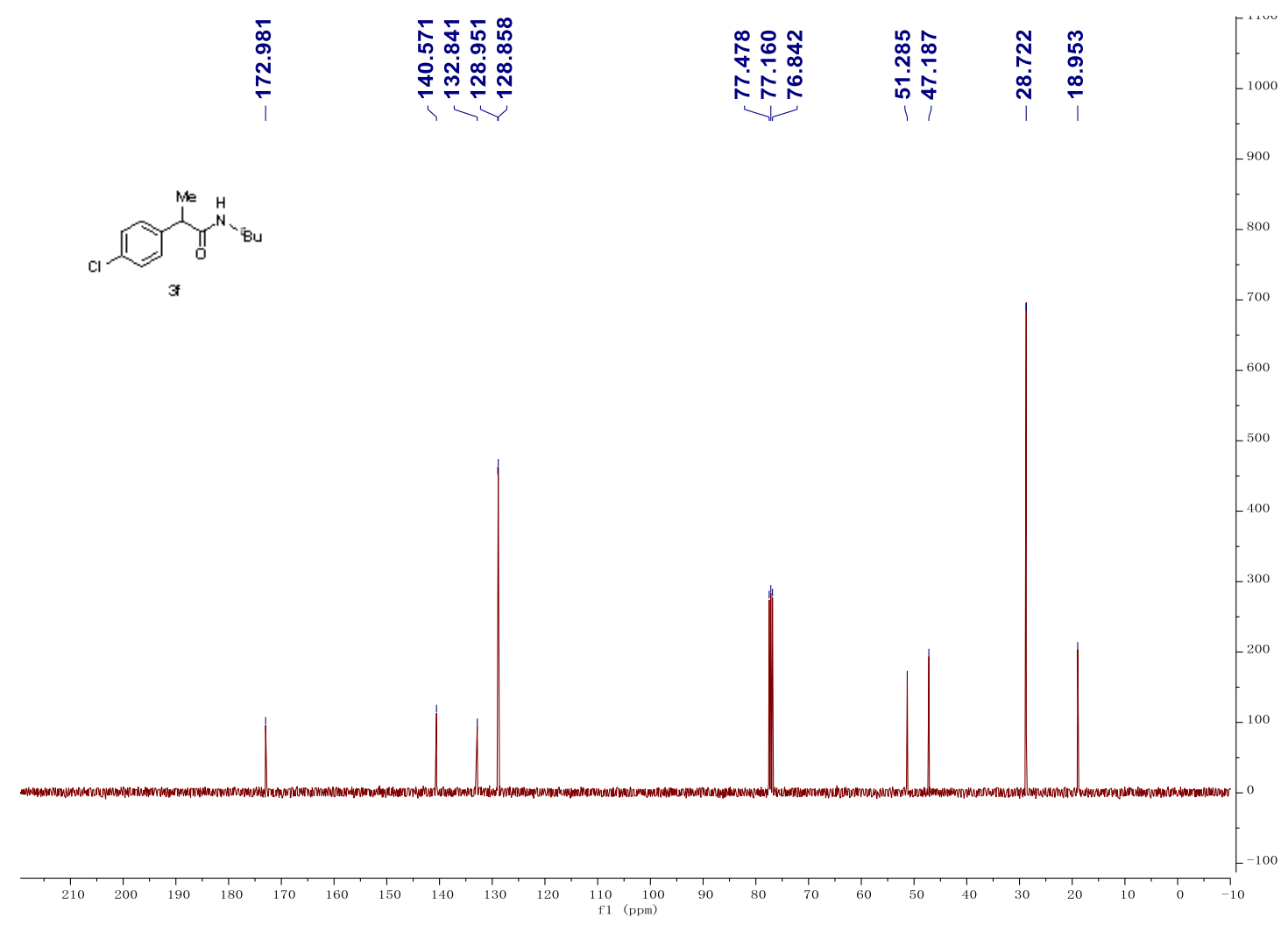


${ }^{1} \mathrm{H}$ NMR-spectrum(400 $\left.\mathrm{MHz}, \mathrm{CDCl}_{3}\right)$ of $3 \mathrm{~g}$

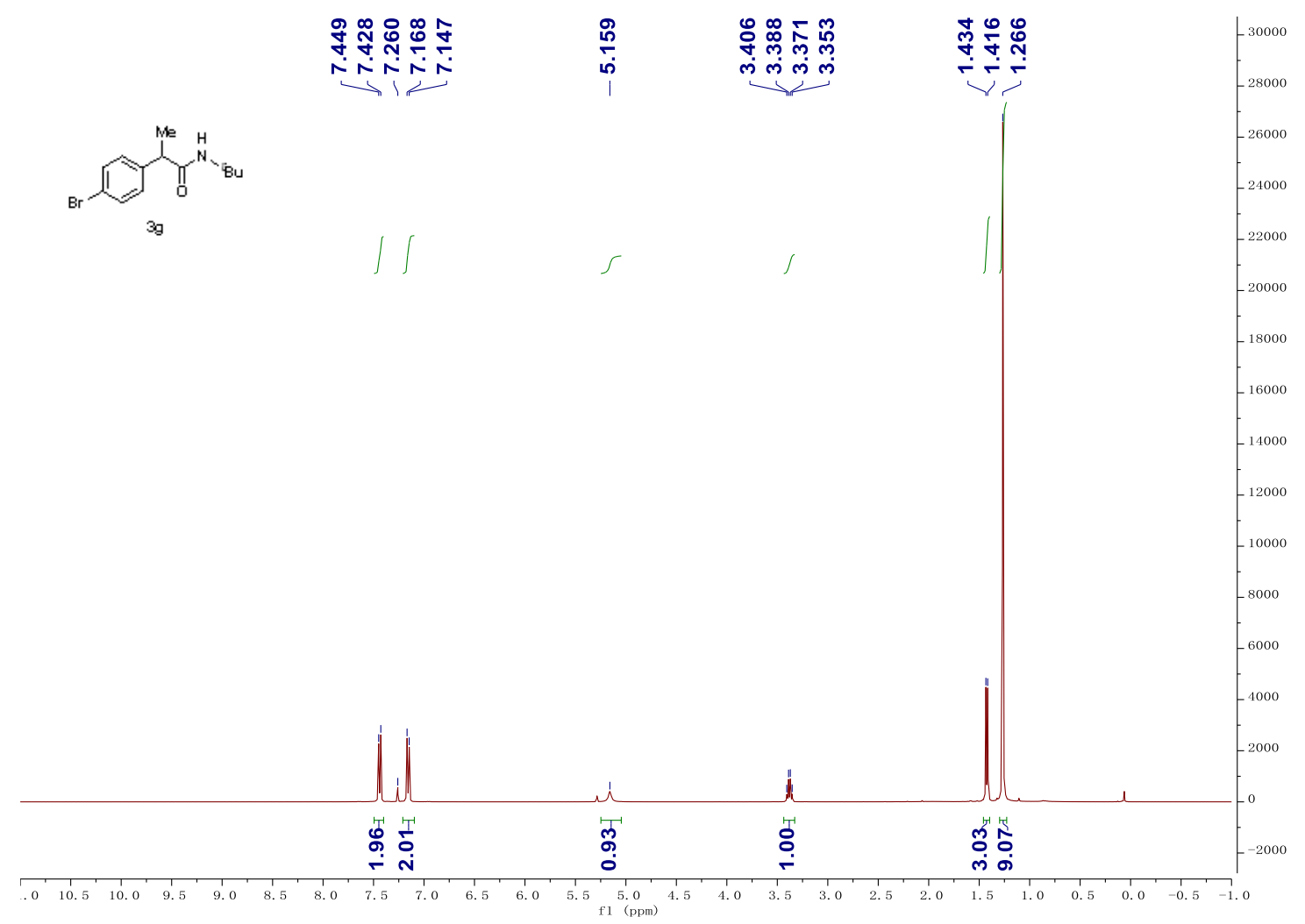

${ }^{13} \mathrm{C}$ NMR-spectrum(100 $\left.\mathrm{MHz}, \mathrm{CDCl}_{3}\right)$ of $3 \mathrm{~g}$

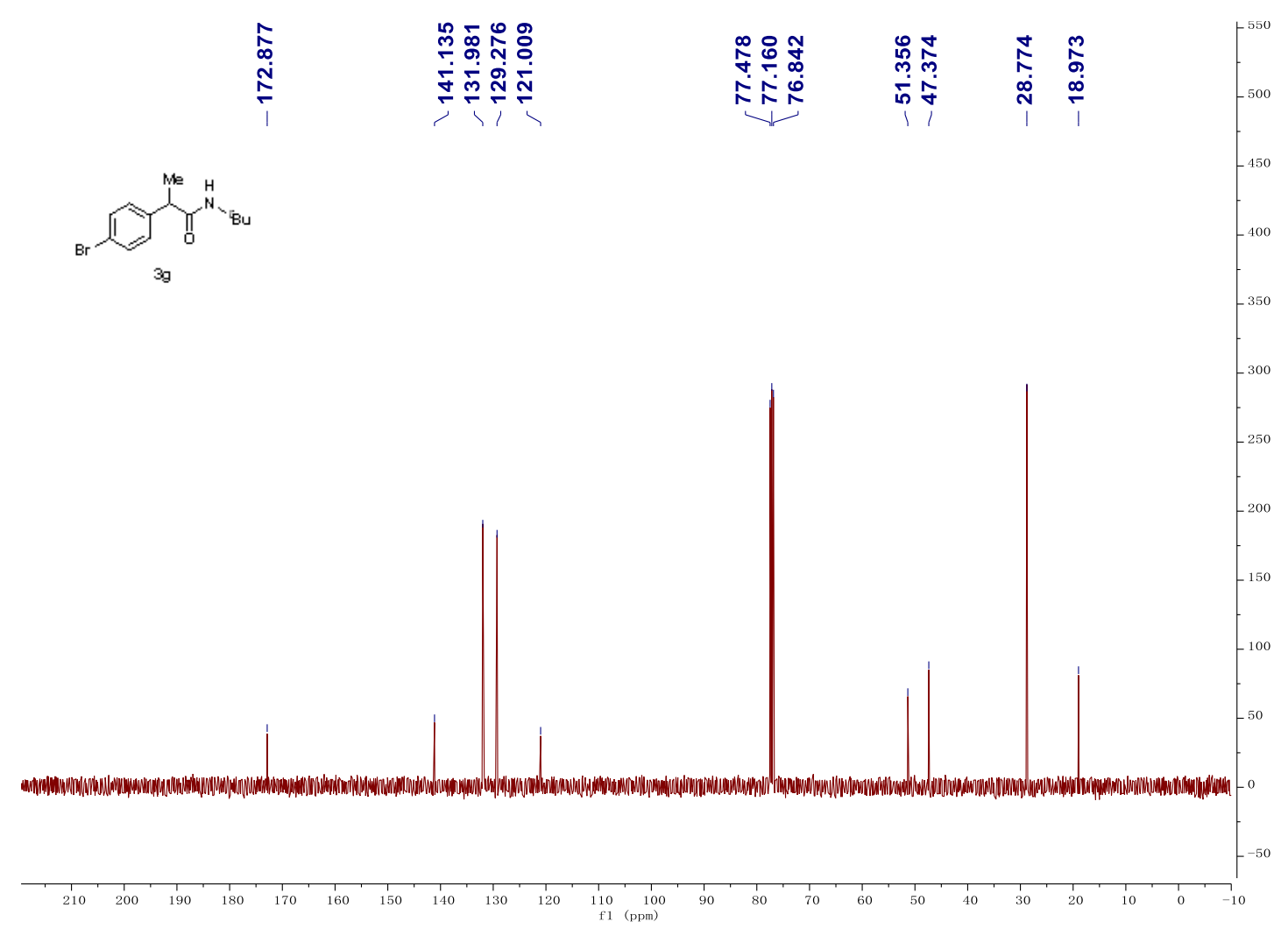


${ }^{1} \mathrm{H}$ NMR-spectrum(400 $\left.\mathrm{MHz}, \mathrm{CDCl}_{3}\right)$ of $3 \mathrm{~h}$

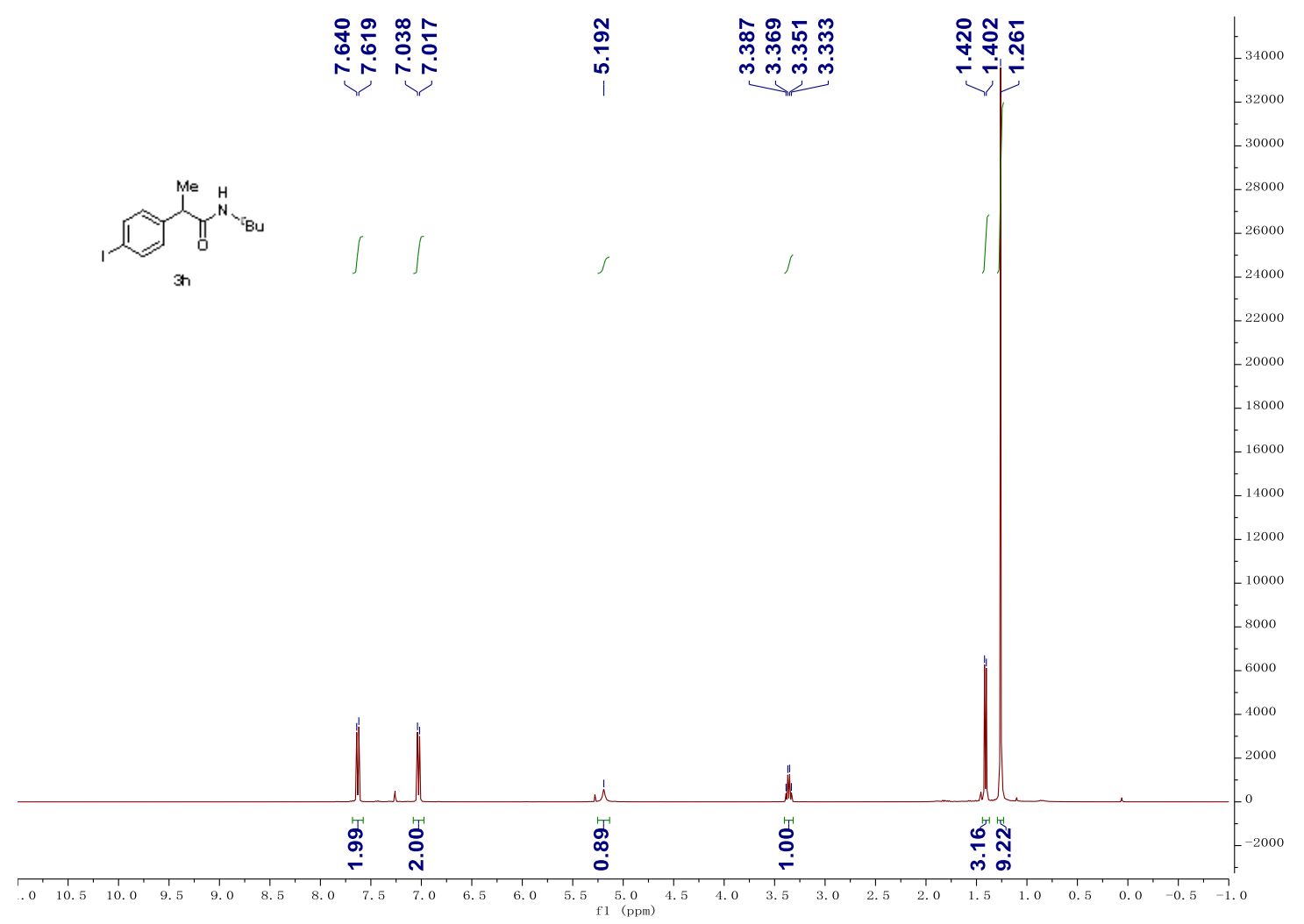

${ }^{13} \mathrm{C}$ NMR-spectrum(100 $\left.\mathrm{MHz}, \mathrm{CDCl}_{3}\right)$ of $3 \mathrm{~h}$

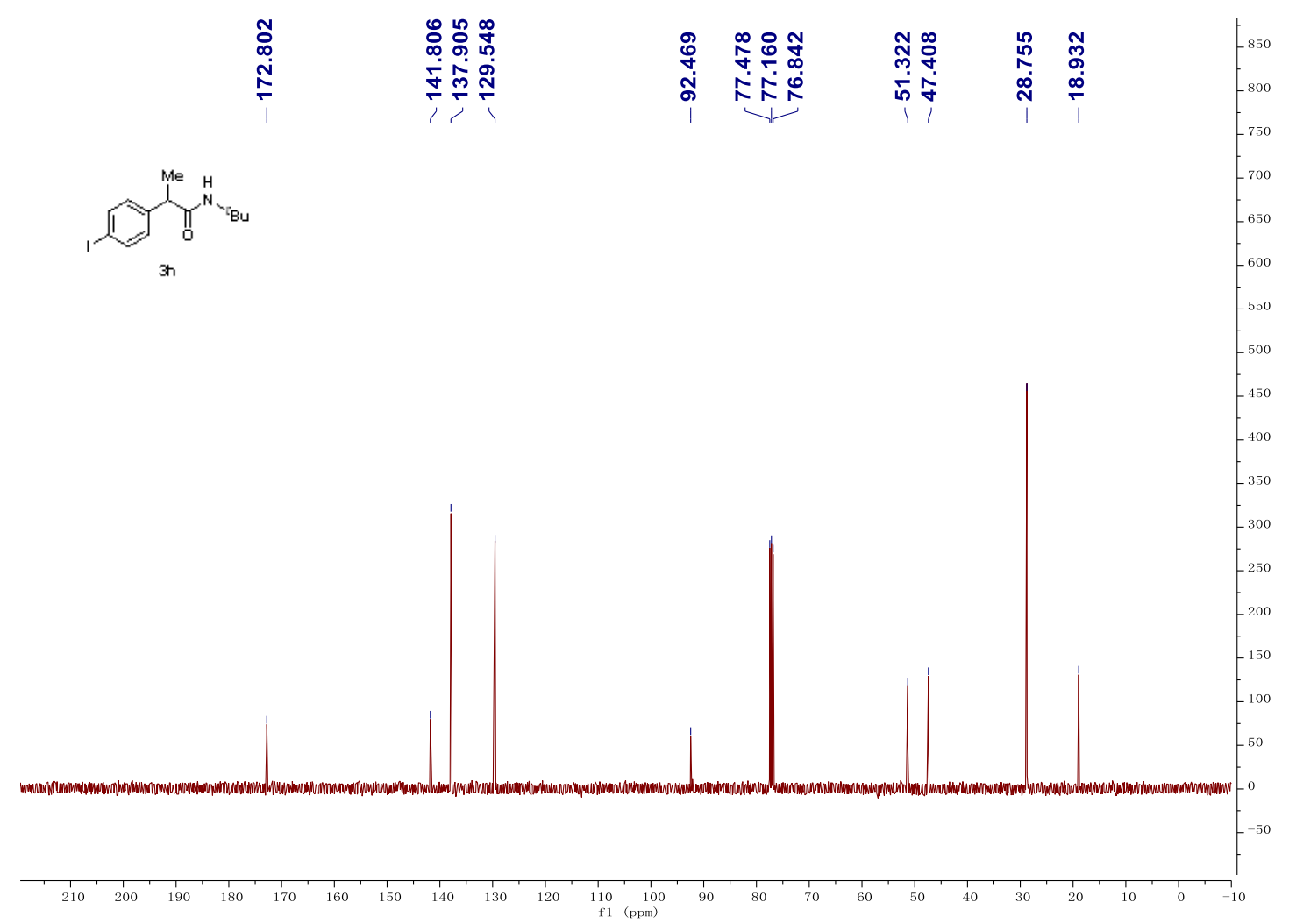


${ }^{1} \mathrm{H}$ NMR-spectrum(400 $\left.\mathrm{MHz}, \mathrm{CDCl}_{3}\right)$ of $3 \mathrm{i}$

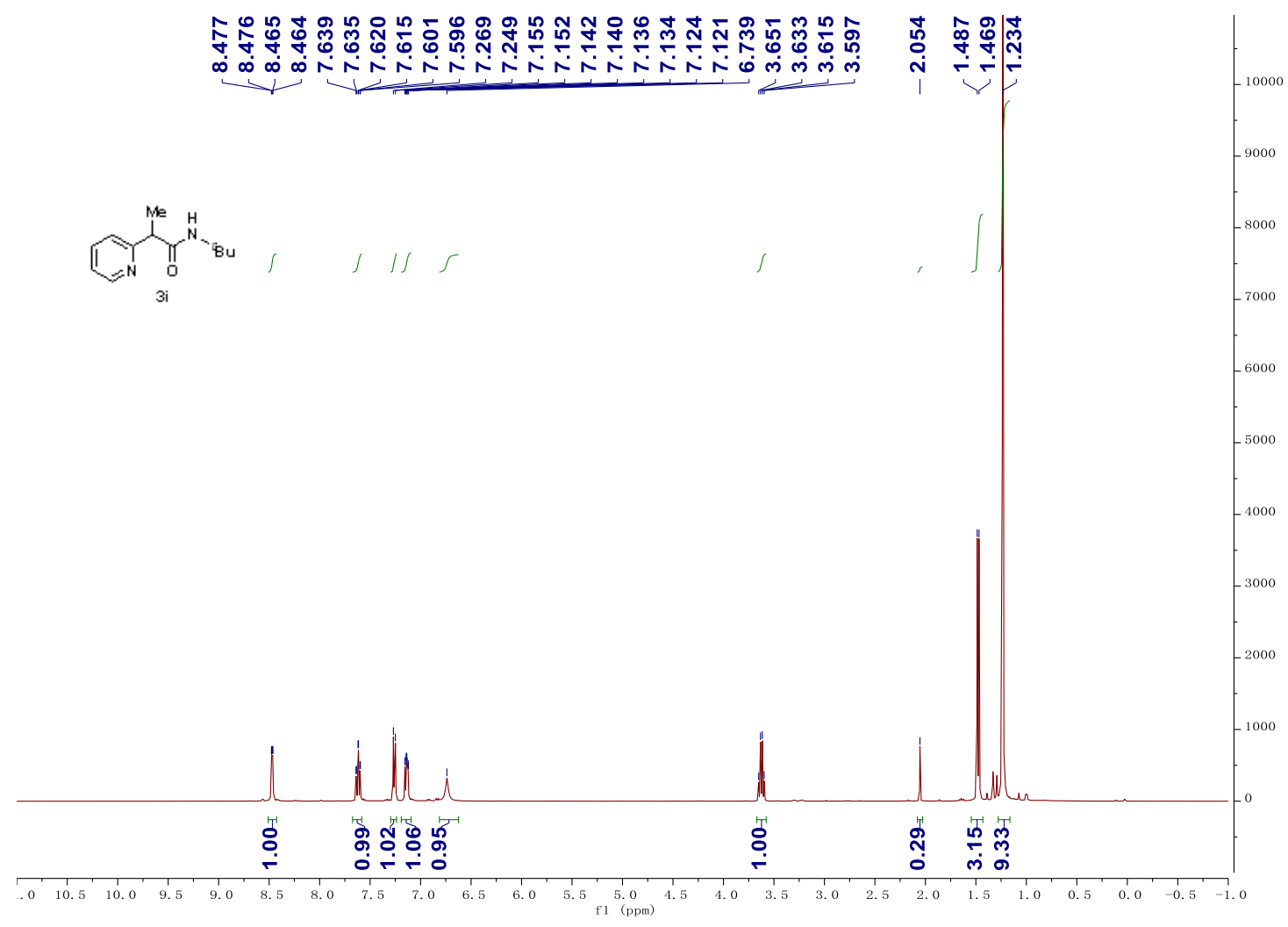

${ }^{13} \mathrm{C}$ NMR-spectrum(100 MHz, $\left.\mathrm{CDCl}_{3}\right)$ of $3 \mathrm{i}$

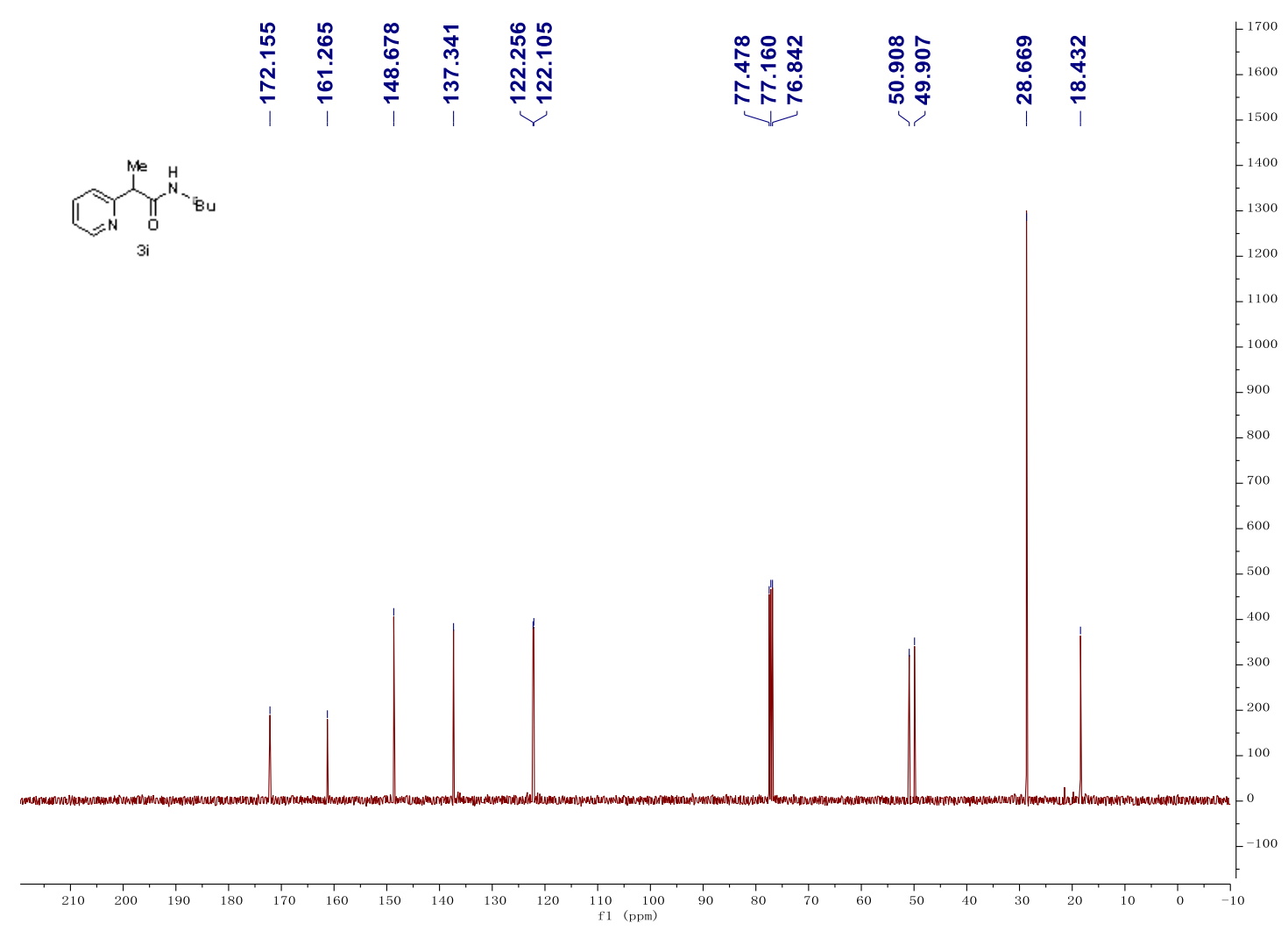


${ }^{1} \mathrm{H}$ NMR-spectrum(400 $\left.\mathrm{MHz}, \mathrm{CDCl}_{3}\right)$ of $3 \mathrm{j}$

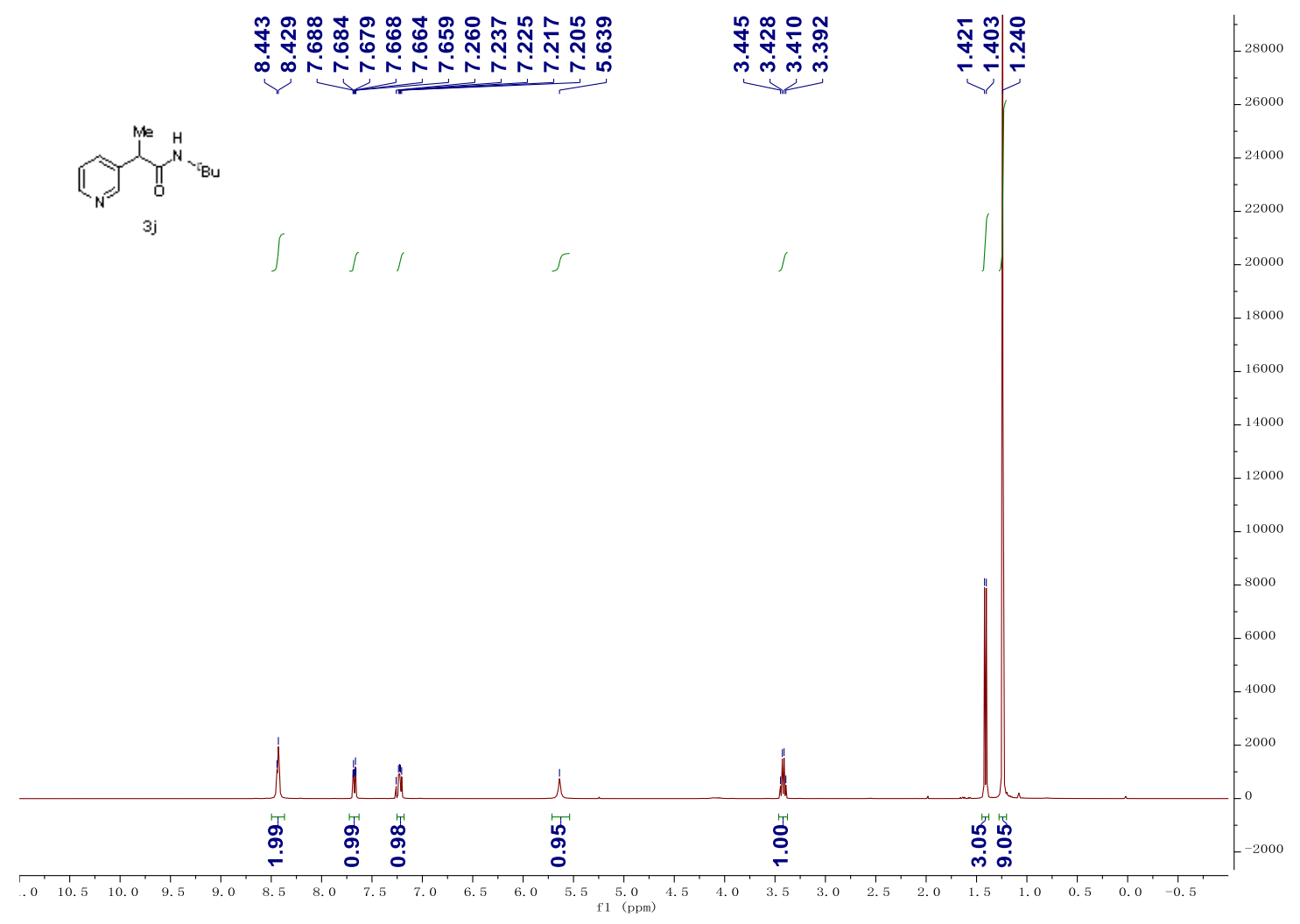

${ }^{13} \mathrm{C}$ NMR-spectrum(100 MHz, $\left.\mathrm{CDCl}_{3}\right)$ of $3 \mathrm{j}$

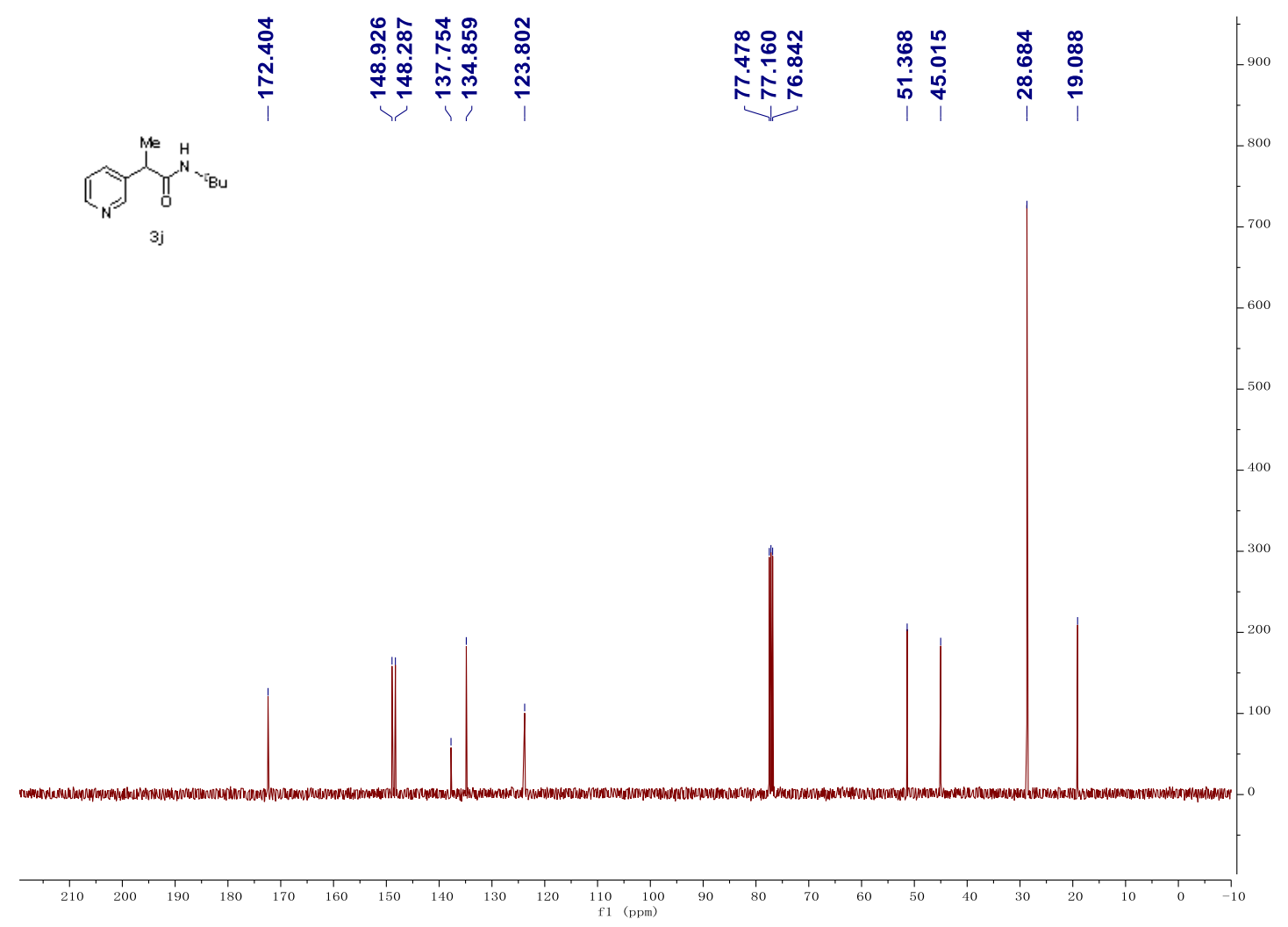


${ }^{1} \mathrm{H}$ NMR-spectrum(400 $\left.\mathrm{MHz}, \mathrm{CDCl}_{3}\right)$ of $3 \mathrm{k}$

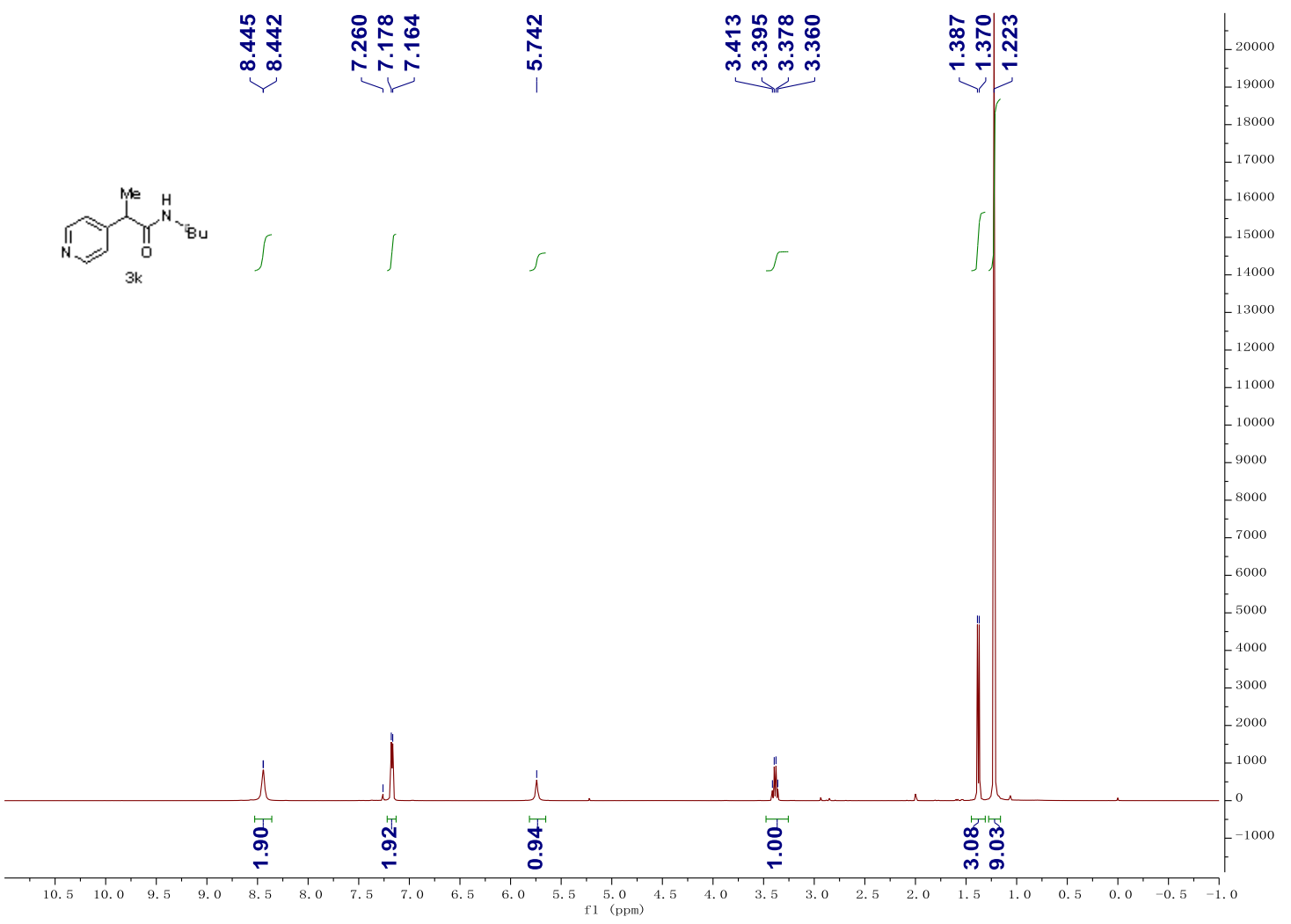

${ }^{13} \mathrm{C}$ NMR-spectrum(100 MHz, $\left.\mathrm{CDCl}_{3}\right)$ of $3 \mathrm{k}$

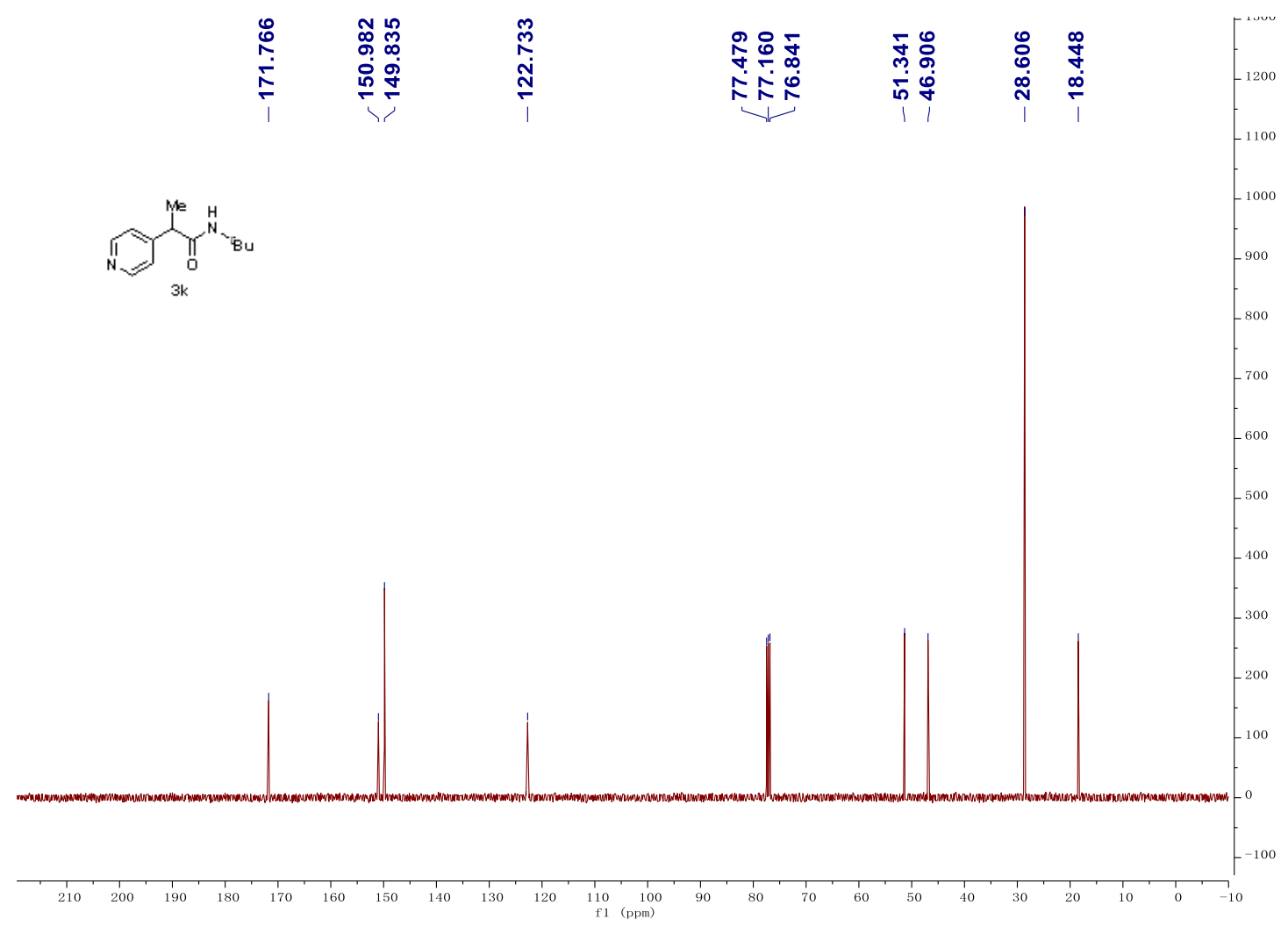


${ }^{1} \mathrm{H}$ NMR-spectrum(400 $\left.\mathrm{MHz}, \mathrm{CDCl}_{3}\right)$ of 31

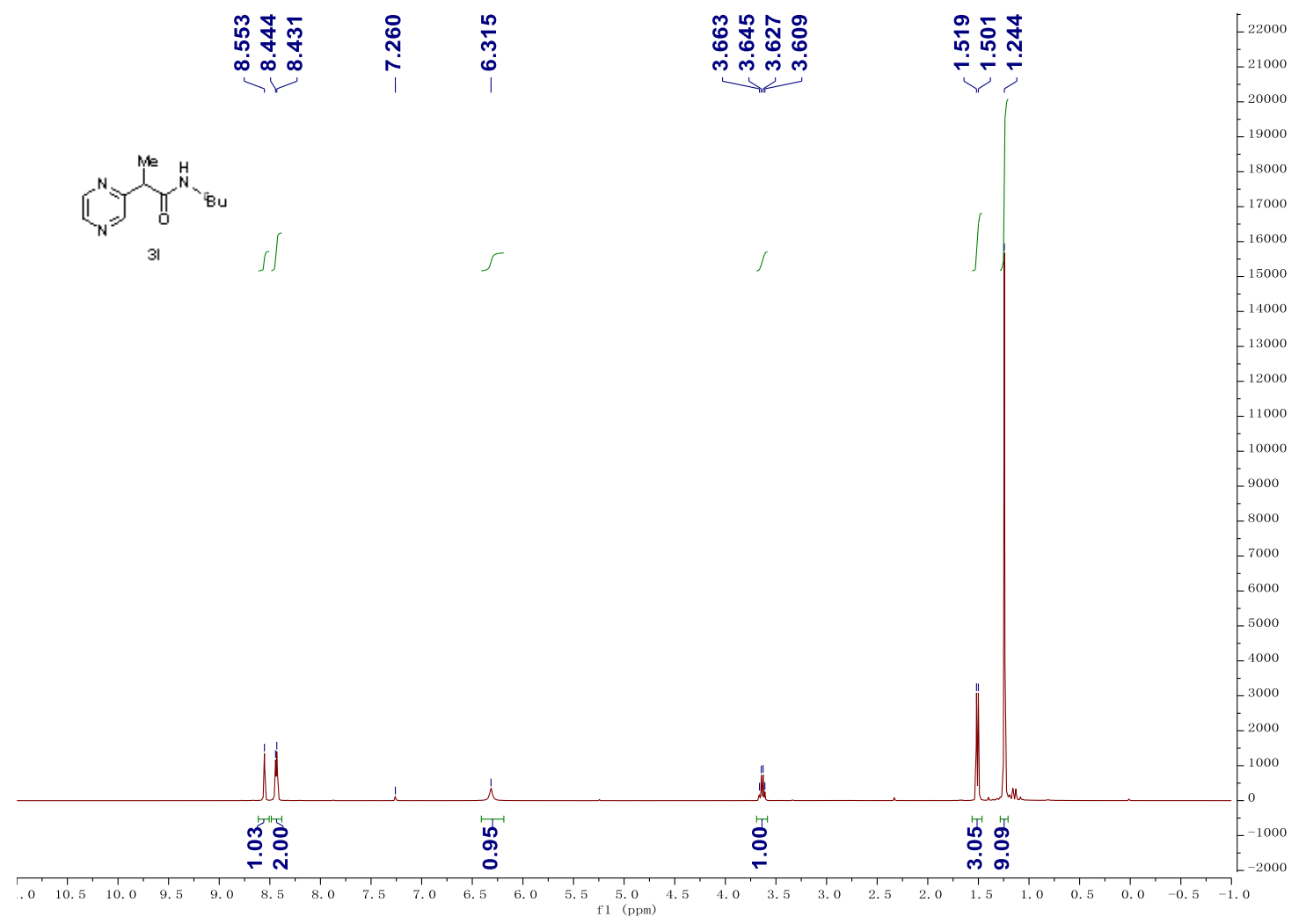

${ }^{13} \mathrm{C}$ NMR-spectrum(100 MHz, $\left.\mathrm{CDCl}_{3}\right)$ of 31

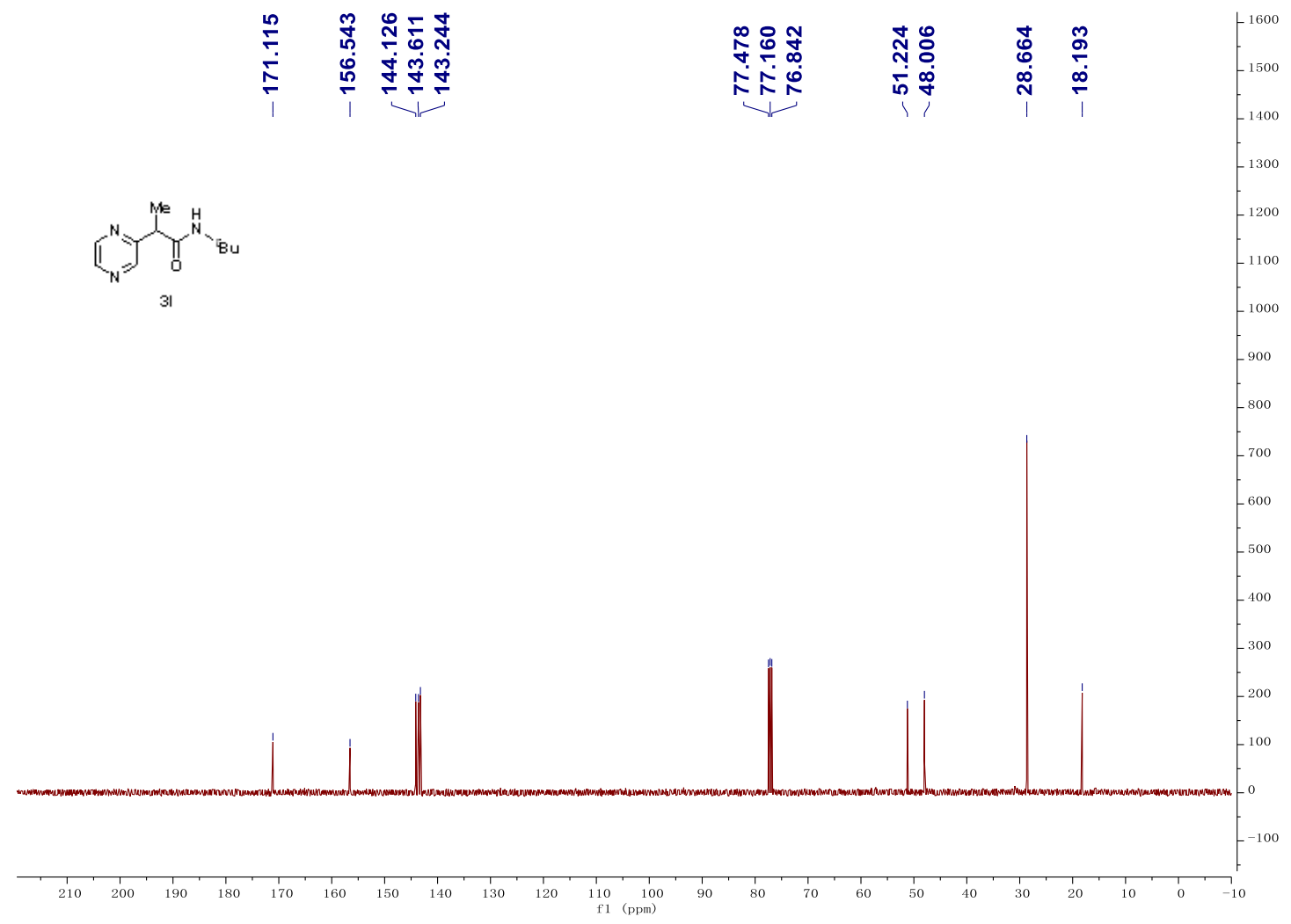


${ }^{1} \mathrm{H}$ NMR-spectrum(400 MHz, $\left.\mathrm{CDCl}_{3}\right)$ of $3 \mathrm{~m}$

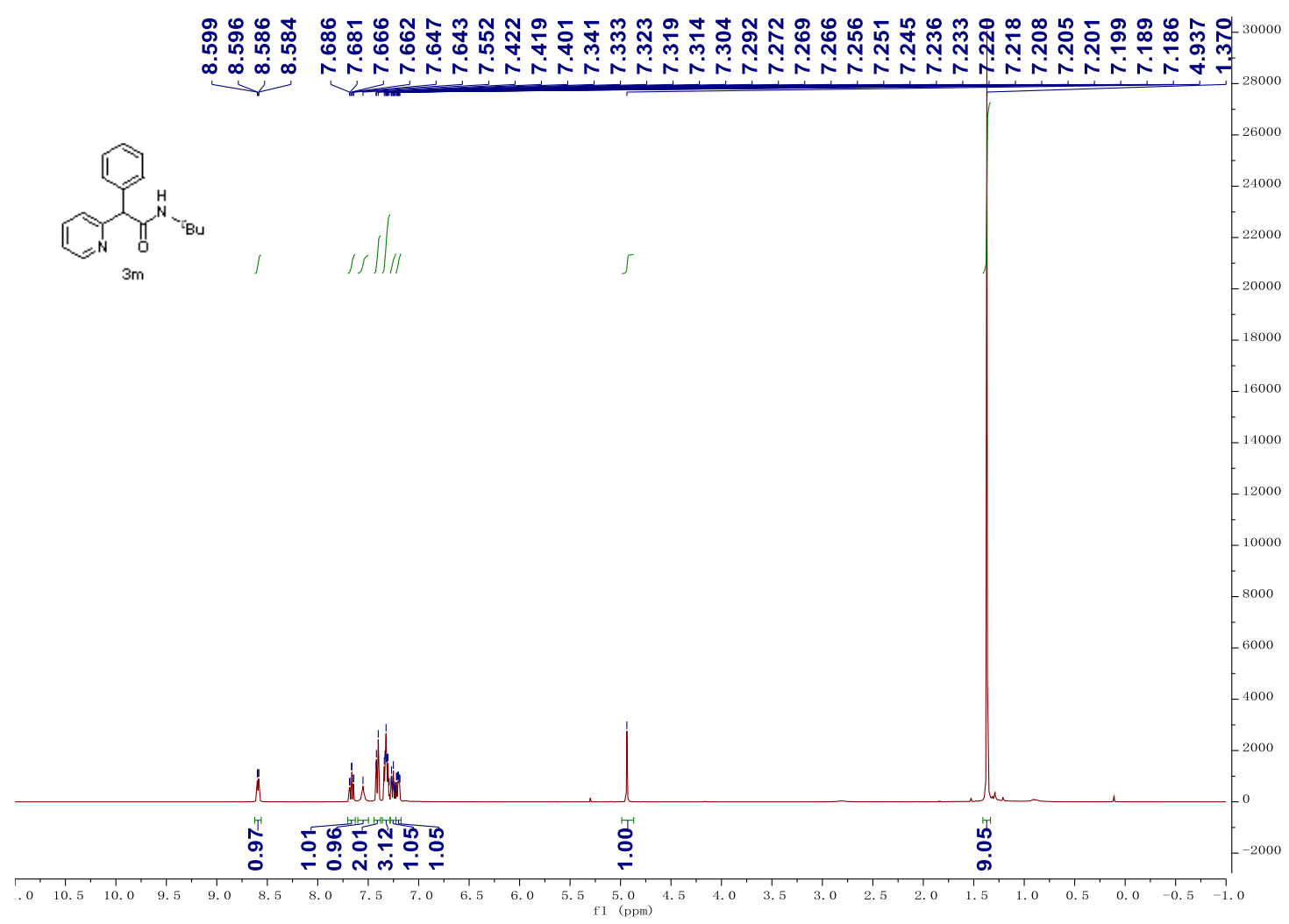

${ }^{13} \mathrm{C}$ NMR-spectrum(100 $\left.\mathrm{MHz}, \mathrm{CDCl}_{3}\right)$ of $3 \mathrm{~m}$

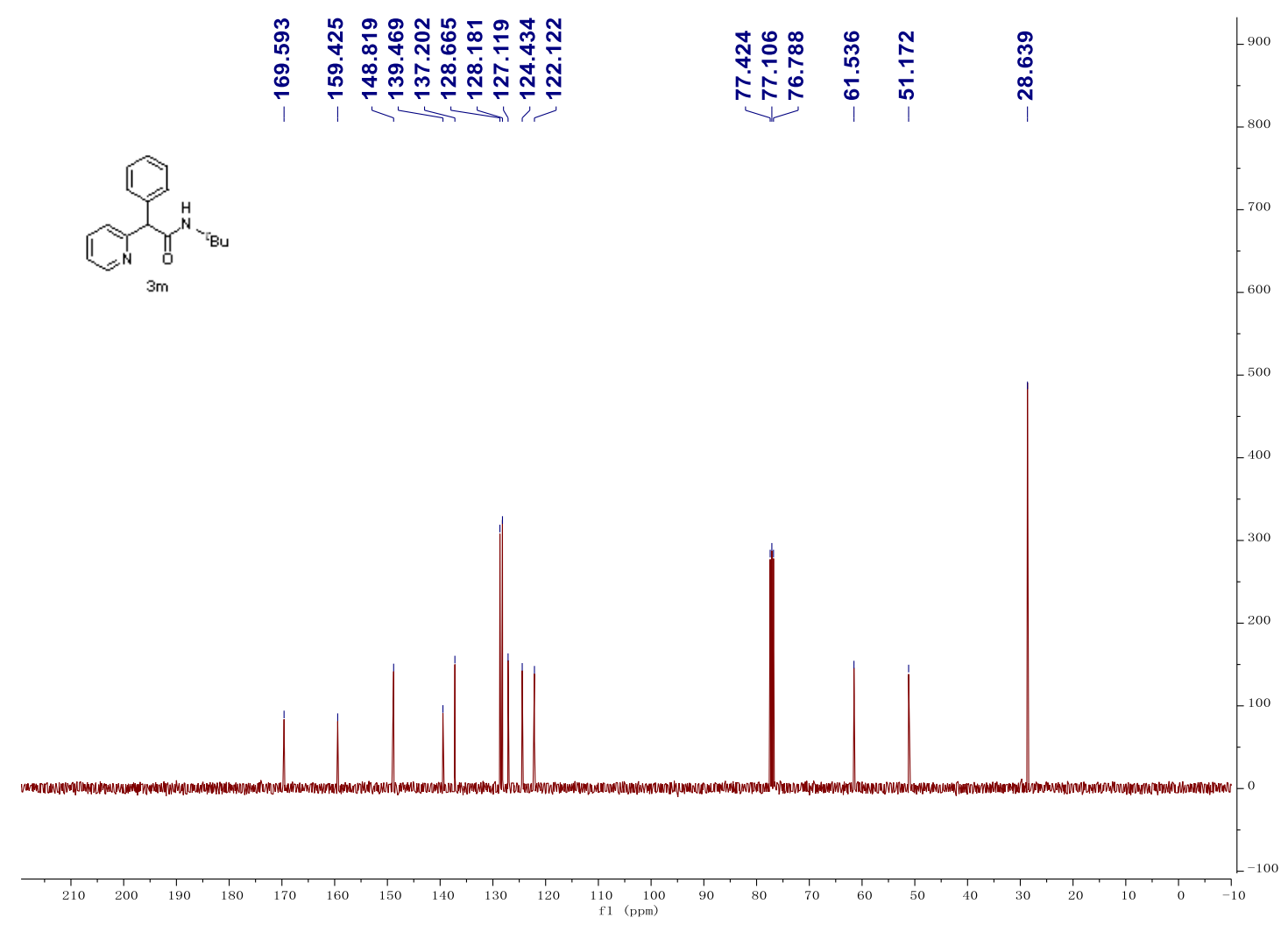


${ }^{1} \mathrm{H}$ NMR-spectrum(400 $\left.\mathrm{MHz}, \mathrm{CDCl}_{3}\right)$ of $3 \mathrm{n}$

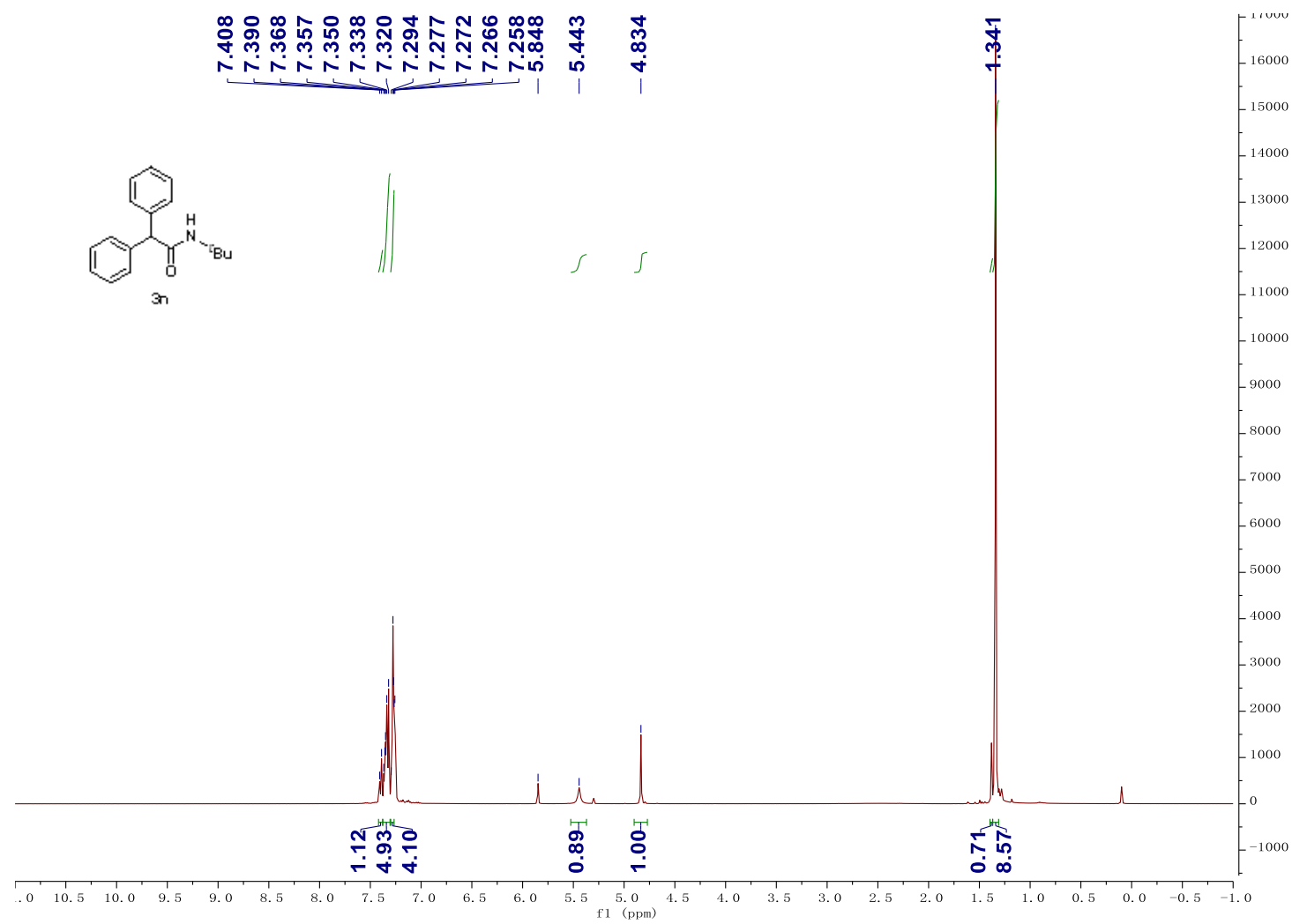

${ }^{13} \mathrm{C}$ NMR-spectrum(100 $\left.\mathrm{MHz}, \mathrm{CDCl}_{3}\right)$ of $3 \mathrm{n}$

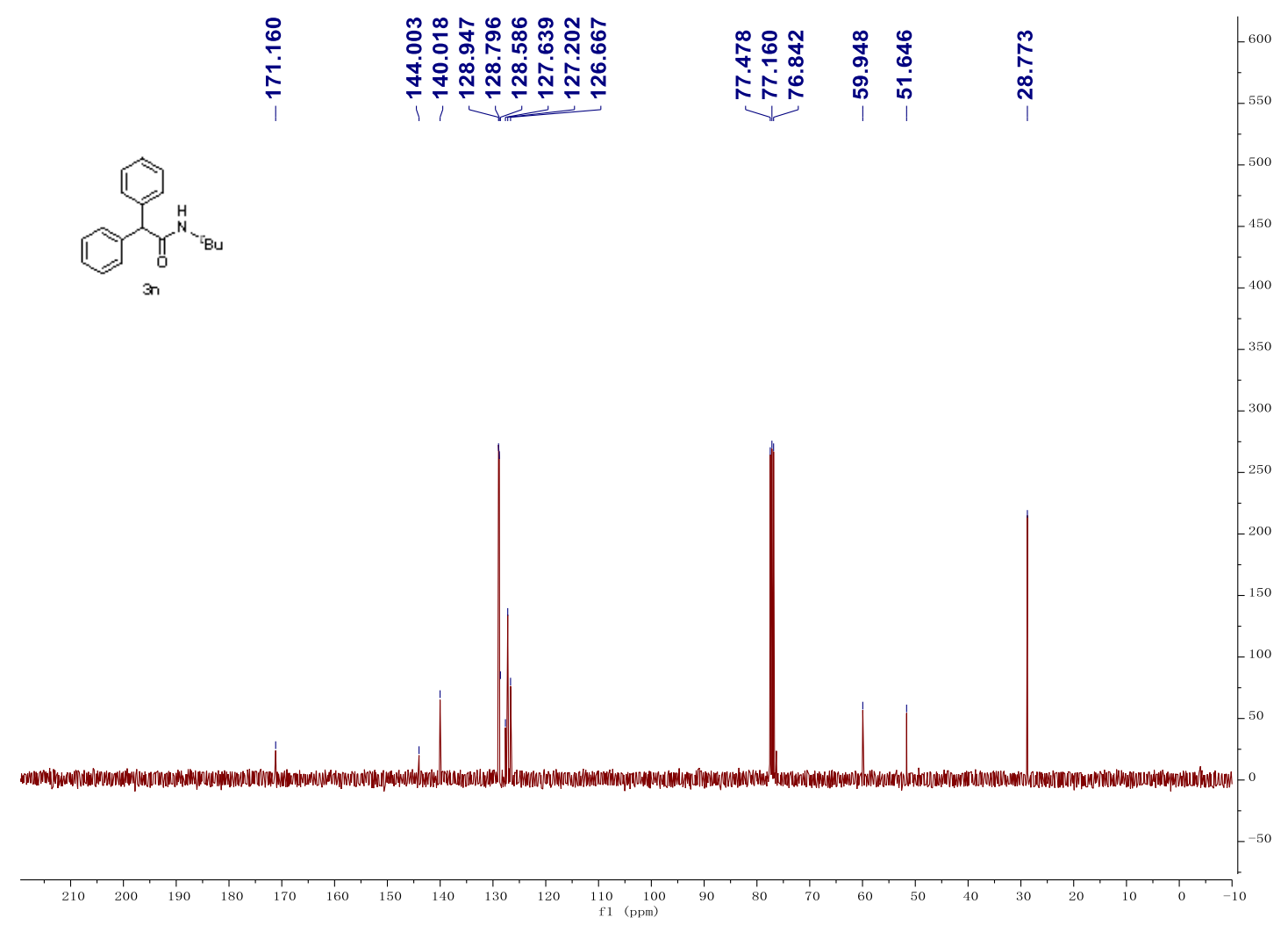


${ }^{1} \mathrm{H}$ NMR-spectrum(400 $\left.\mathrm{MHz}, \mathrm{CDCl}_{3}\right)$ of 30

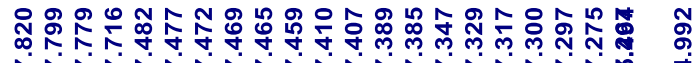

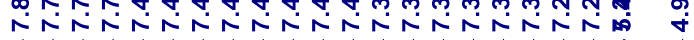

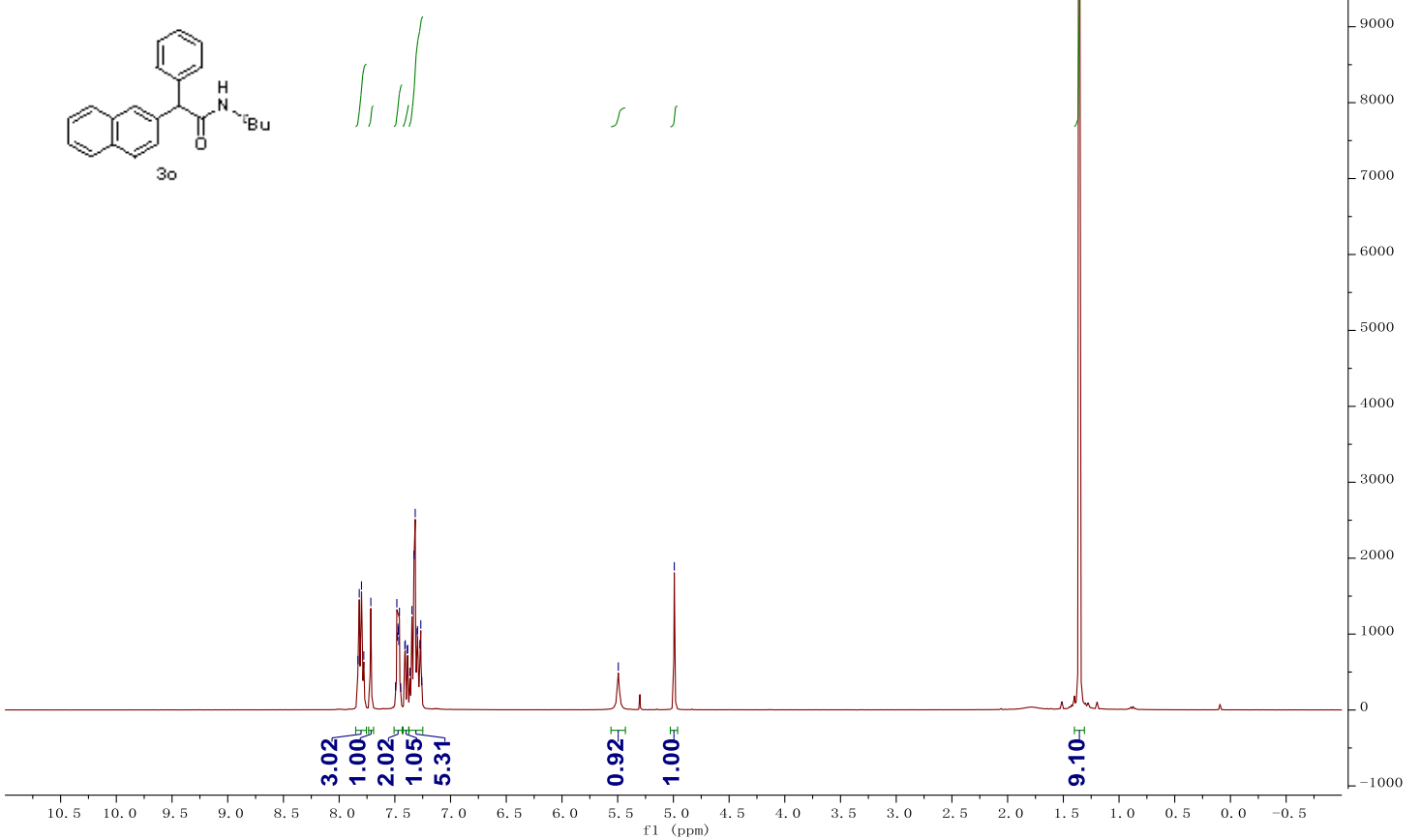

${ }^{13} \mathrm{C}$ NMR-spectrum(100 $\left.\mathrm{MHz}, \mathrm{CDCl}_{3}\right)$ of 30

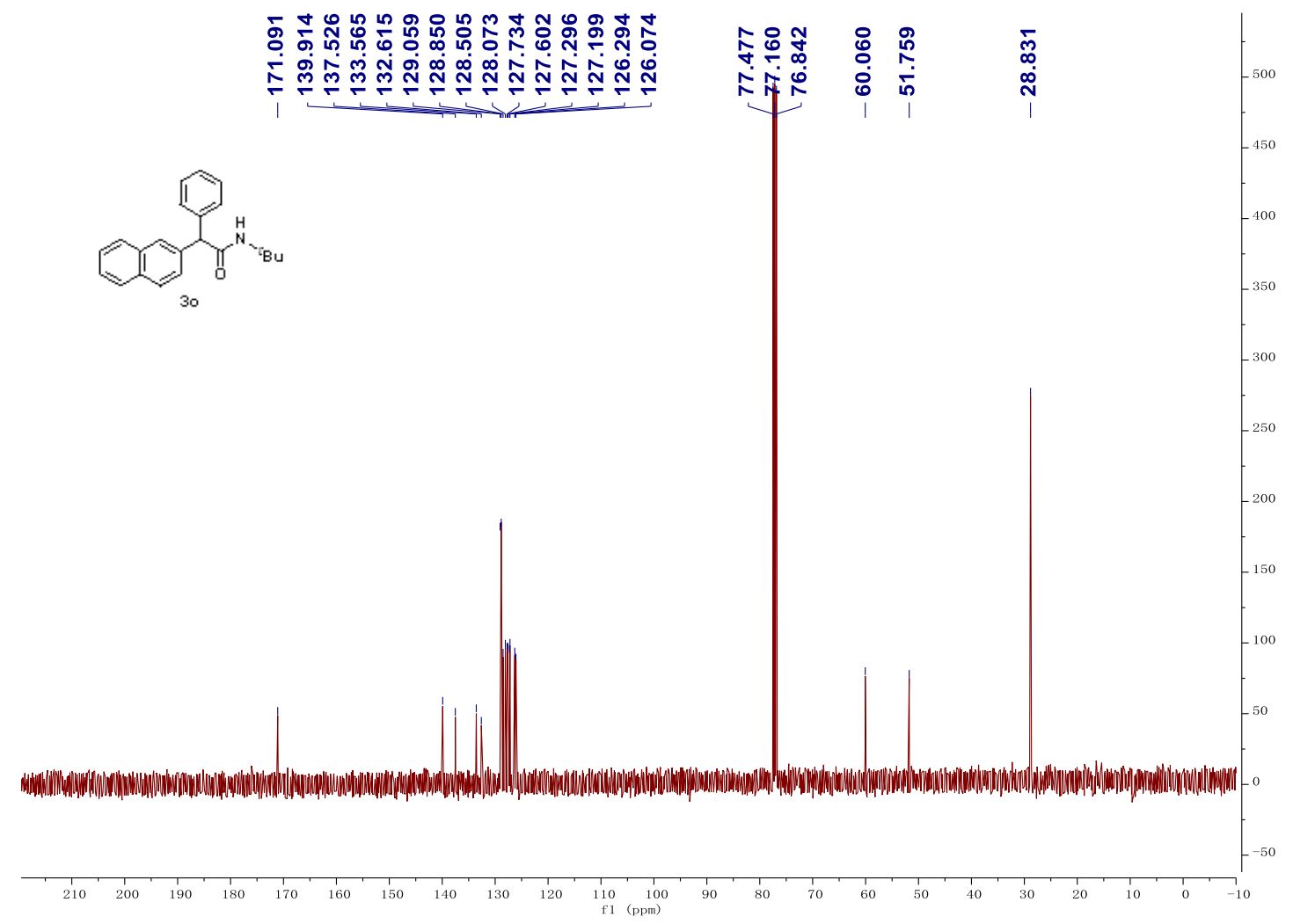


${ }^{1} \mathrm{H}$ NMR-spectrum(400 $\left.\mathrm{MHz}, \mathrm{CDCl}_{3}\right)$ of 3p

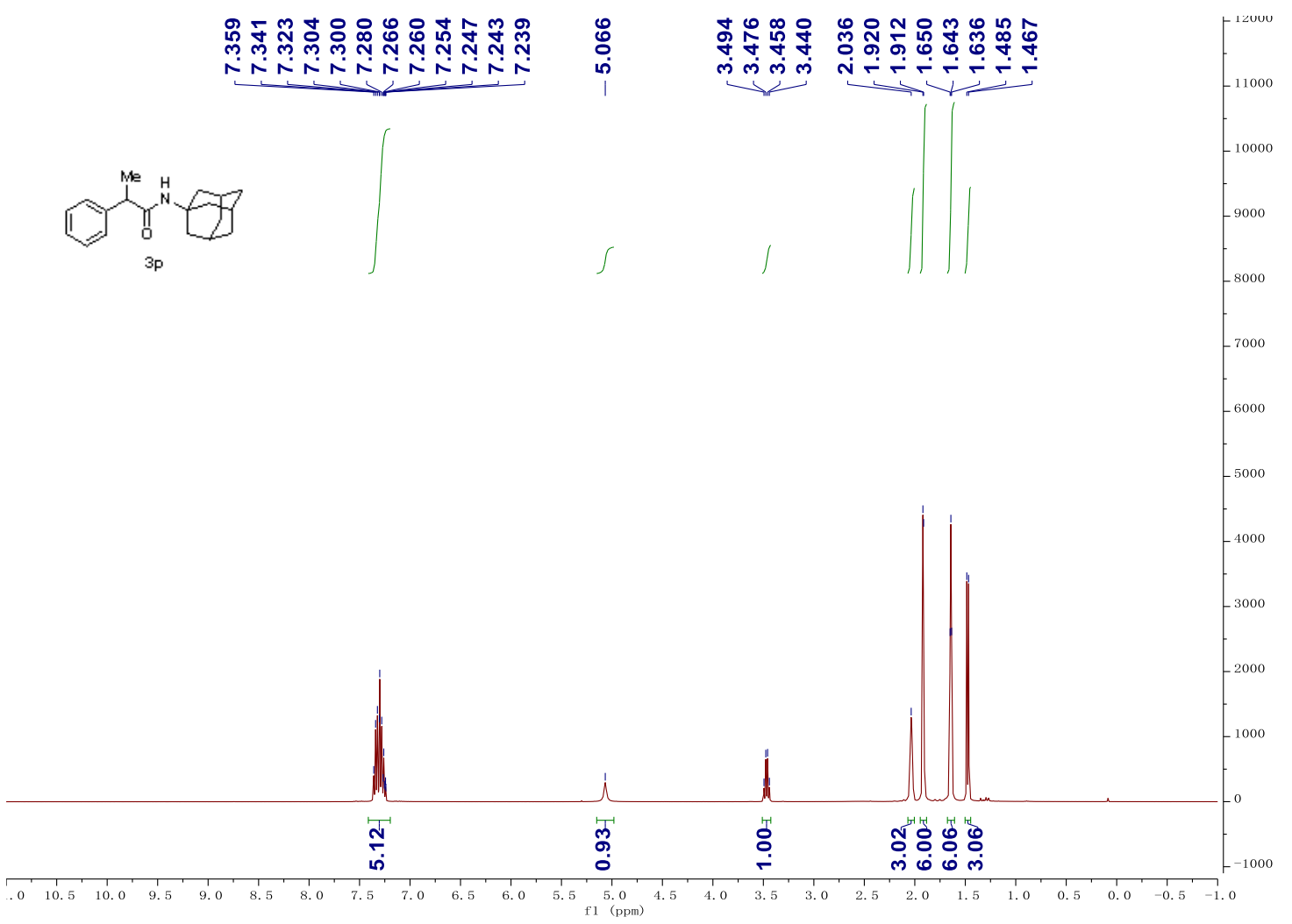

${ }^{13} \mathrm{C}$ NMR-spectrum(100 $\left.\mathrm{MHz}, \mathrm{CDCl}_{3}\right)$ of 3p

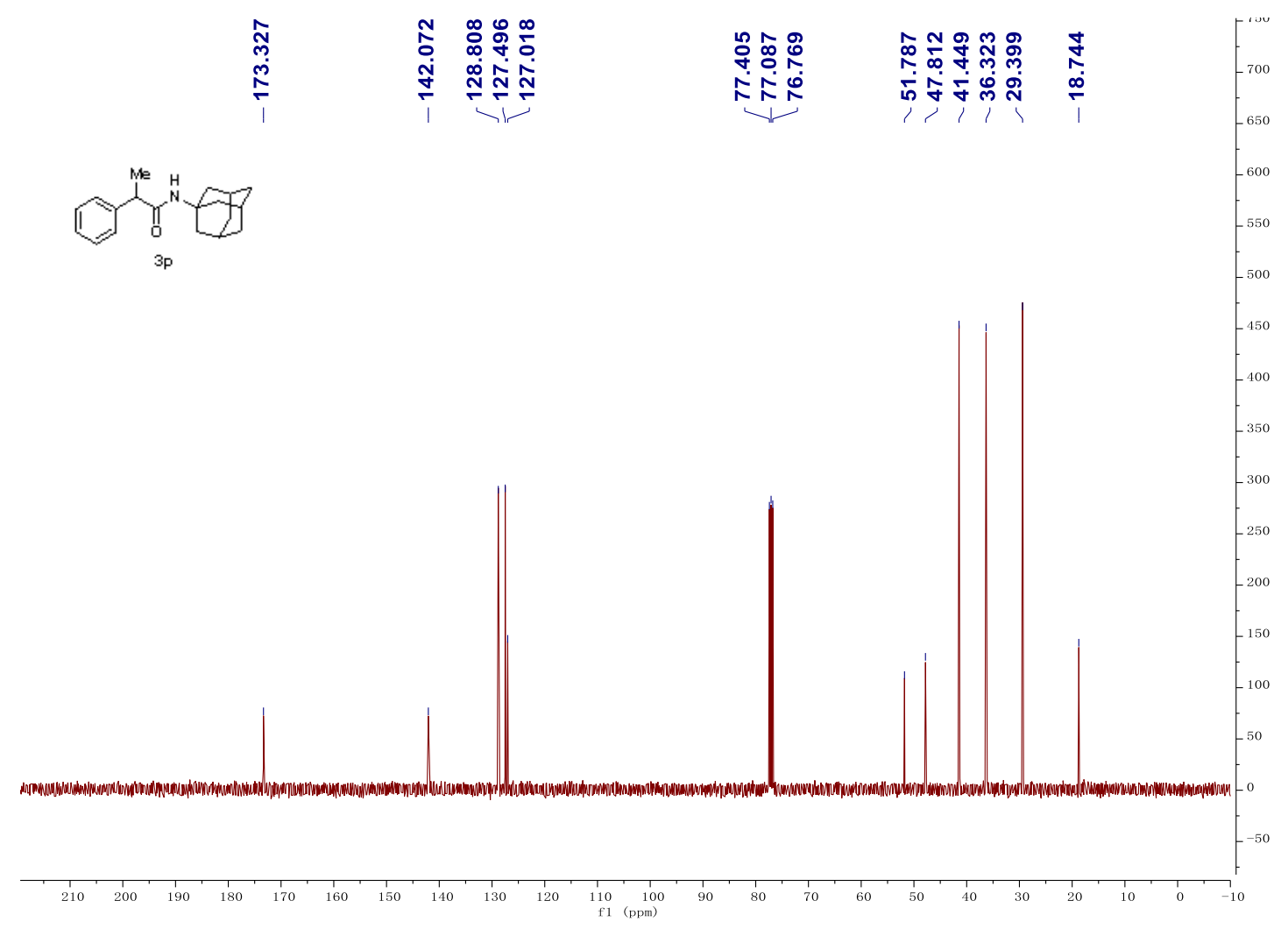




\section{References}

[1] Amemiya, Y.; Hong, S. S.; Venkataraman, B. V.; Patil, P. N.; Shams, G.; Romstedt, K.; Feller, D. R.; Hsu, F. L.; Miller, D. D. Synthesis and a-Adrenergic Activities of 2- and 4-Substituted Imidazoline and Imidazole Analogs. J. Med. Chem. 1992, 35, 750-755.

[2] Iwamoto, H.; Endo, K.; Ozawa, Y.; Watanabe, Y.; Kubota, K.; Imamoto, T.; Ito, H. Copper(I)Catalyzed Enantioconvergent Borylation of Racemic Benzyl Chlorides Enabled by Quadrant-byquadrant Structure Modification of Chiral Bisphosphine Ligands. Angew. Chem. Int. Ed. 2019, 58, 11112-11117.

[3] Alapafuja, S. O.; Malamas, M. S.; Shukla, V.; Zvonok, A.; Miller, S.; Daily, L.; Rajarshi, G.; Miyabe, C. Y.; Chandrashekhar, H.; Wood, J.; Tyukhtenko, S.; Straiker, A.; Makriyannis, A. Synthesis and Evaluation of Potent and Selective MGL Inhibitors as a Glaucoma Treatment. Bioorg. Med. Chem. 2019, 27, 55-64.

[4] Cherney, A. H.; Kadunce, N. T.; Reisman, S. E. Catalytic Asymmetric Reductive Acyl Crosscoupling: Synthesis of Enantioenriched Acyclic $\alpha, \alpha$-Disubstituted Ketones. J. Am. Chem. Soc. 2013, 135, 7442-7445.

[5] Yuho, T.; Yoshiaki, K.; Masami, S.; Takahiro, F.; Yasuhide, Y. The Substituent Effect. VIII. Solvolysis of $m$ - and $p$-Substituted $\alpha$-Methylbenzyl Chlorides. Bull. Chem. Soc. Jpn, 1975, 48, 3337-3346.

[6] Magubane, M. N.; Alam, M. G.; Ojwach, S. O.; Munro, O. Q. Orientation towards Asymmetric Transfer Hydrogenation of Ketones Catalyzed by (Pyrazolyl)ethyl)pyridine $\mathrm{Fe}(\mathrm{II})$ and $\mathrm{Ni}$ (II) Complexes. J. Mol. Struct. 2017, 1135, 197-201.

[7] Sainsbury, M.; Schinazi, R. F. Chemistry of 6H-Pyrido[4,3-b]carbazoles. Part V. A Simple Synthe of Ellipticines. J. Chem. Soc., Perkin Trans. 1, 1976, 1155-1160.

[8] Liu, J.; Zhang, X.; Yi, H.; Liu, C.; Liu, R.; Zhang, H.; Zhuo, K.; Lei, A. Chloroacetate - Promoted Selective Oxidation of Heterobenzylic Methylenes under Copper Catalysis. Angew. Chem. Int. Ed. 2014, 54, 1261-1265.

[9] Chern, J.-H.; Shia, K.-S.; Hsu, T.-A.; Tai, C.-L.; Lee, C.-C.; Lee, Y.-C.; Chang, C.-S.; Tseng, S.N.; Shih, S.-R. Design, Synthesis, and Structure-activity Relationships of Pyrazolo[3,4d]pyrimidines: A Novel Class of Potent Enterovirus Inhibitors. Bioorg. Med. Chem. Lett. 2004, $14,2519-2525$.

[10] Labeeuw, O.; Levoin, N.; Billot, X.; Danvy, D.; Calmels, T.; Krief, S.; Ligneau, X.; BerrebiBertrand, I.; Robert, P.; Lecomte, J. M.; Schwartz, J. C.; Capet, M. Synthesis and Evaluation of A 2-Benzothiazolylphenylmethyl Ether Class of Histamine H4 Receptor Antagonists. Bioorg. Med. Chem. Lett. 2016, 26, 5263-5266.

[11]Savela, R.; Wärnå, J.; Murzinb, D. Y.; Leino, R. Iron Catalyzed Halogenation of Benzylic Aldehydes and Ketones. Catal. Sci. Technol. 2015, 5, 2406-2417.

[12]Wu, S.; Huang, J.; Gazzarrini, S.; He, S.; Chen, L.; Li, J.; Xing, L.; Li, C.; Chen, L.; Neochoritis, C. G.; Liao, G. P.; Zhou, H.; Domling, A.; Moroni, A.; Wang, W. Isocyanides as Influenza A Virus Subtype H5N1 Wild-type M2 Channel Inhibitors. ChemMedChem. 2015, 10, 1837-1845.

[13] Li, J.; Liu, Y.; Li, C.; Jia, X. Syntheses of Spirocyclic Oxindole-Butenolides by Using Three- 
Component Cycloadditions of Isocyanides, Allenoates, and Isatins. Chem. -Eur. J. 2011, 17, 7409-7413.

[14] Bon, R. S.; Hong, C.; Bouma, M. J.; Schmitz, R. F.; de Kanter, F. J. J.; Lutz, M.; Spek, A. L.; Orru. R. V. A. Novel Multicomponent Reaction for the Combinatorial Synthesis of 2-Imidazolines. Org. Lett. 2003, 5, 3759-3762.

[15] Zhou, F.; Ding, K.; Cai, Q. Palladium-Catalyzed Amidation of $N$-Tosylhydrazones with Isocyanides. Chem. -Eur. J. 2011, 17, 12268-12271.

[16] Shiina, I.; Kawakita, Y. The Effective Use of Substituted Benzoic Anhydrides for the Synthesis of Carboxamides. Tetrahedron 2004, 60, 4729-4733. 